\title{
Franz Kreuter
}

\section{Beitrag zur Berechnung uno flusfübrung Der Staumauern}

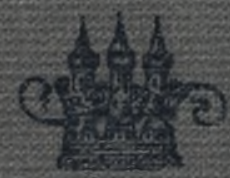

IIfindjen und Berlin Oruck unio Derlag von R. Olbenbourg 


\section{Bıblioteka Politechniki Krakowskıej}

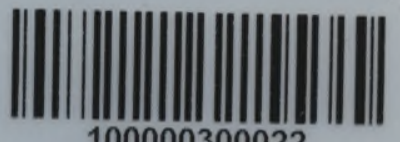

100000300022 




\section{BEITRAG}

zur

\section{Berechnung und Ausführung}

\section{der Staumauern}

von

\section{FRANZ KREUTER}

Ingenieur

Professor an der Technischen Hochschule in München

Mit 20 Abbildungen
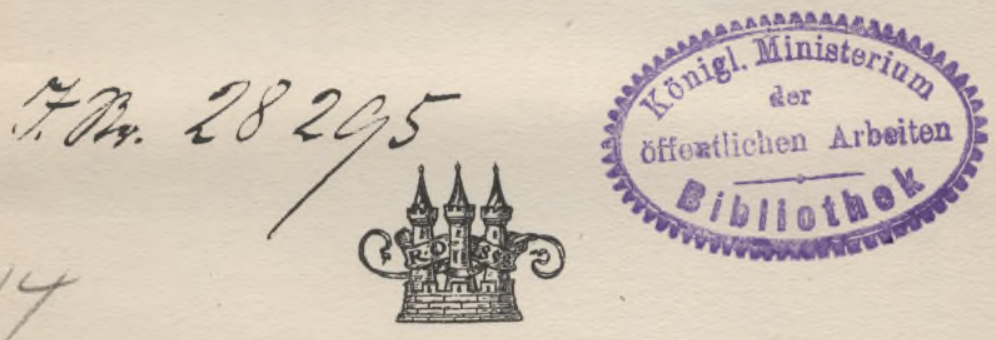

München und Berlin

Druck und Verlag von R. Oldenbourg 1909

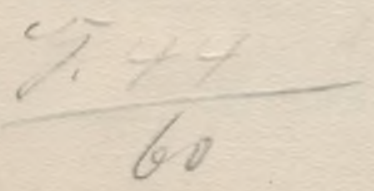


B!BULTEKA POLITECHHICZHA

KRAKÓW

II 31144

Akc. Nr. $\quad 2012 / 49$ 


\section{Inhaltverzeichnis.}

Seite

Vorbemerkung . . . . . . . . . . . . . . 5

I. Praktische Vorbedingungen:

a) Sicherung der Mauer gegen den Auftrieb desWassers 8

b) Grundgestalt des Mauerquerschnittes . . . . . 10

c) Lagerflächen freistehender Staumauern . . . . 12

II. Theoretische Vorbedingungen :

a) Praktische Zulässigkeit der Trapezregel. - Größe der Randspannungen . . . . . . . .

b) Festsetzung der praktischen Grenze für die Beanspruchung des Gemäuers .

III. Entwickelung der Bestimmungsgleichungen:

a) Hypothesen und Bezeichnungen . . . . . . . 23

b) Widerstand gegen das Gleiten längs einer wagerechten Schnittebene .. . . . . . . . . . 24

c) Zusammendrückung an der talseitigen (äußeren) Böschung . . . . . . . . . . . . . . . .

d) Zusammendrückung an der bergseitigen (inneren) Böschung . . . . . . . . . . . . . . . . 26

e) Grenzhöhe oder praktische Grenze für die Gültigkeit des Grunddreieckes . . . . . . . . . .

f) Fall, wo die bergseitige (innere) Wand nicht lotrecht ist. - Notwendigkeit einer inneren Böschung

IV. Anwendung:

A. Abteilung der Querschnittfläche . . . . . . . . 31

B. Berechnung der Abmessungen . . . . . . . . 34

a) Kopf . . . . . . . . . . . . . . . . . . 34

b) Hals . . . . . . . . . . . . . . . . . . 34

c) Rumpf, obere Hälfte . . . . . . . . . . . . 37

d) Rumpf, untere Hälfte .......... . . 44

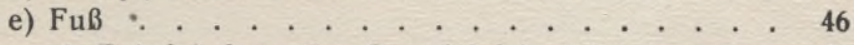

1. Entwickelung der Grundgleichungen ... . 46

2. Ableitung der Bestimmungsgleichungen . . 50

Schlußbemerkungen. . . . . . . . . . . . . . . 53 



\section{Vorbemerkung.}

Seit ich eine erste Berechnung des kleinstmöglichen Querschnittes der Staumauern veröffentlichte ${ }^{1}$ ), haben französische und englische Mathematiker und Ingenieure neue Gesichtspunkte aufgestellt ${ }^{2}$ ).

1) On the Design of Masonry Dams, Min. of proc. of The Institution of Civil Engineers. Vol. CXV. Session 1893-94, part. I, p. 53.

Berechnung der Staumauern. Zeitschr. f. Bauw. 1894, S. 465.

`) Maurice Lévy: Quelques considérations sur la construction des grands barrages. Comptes rendus des séances de l'académie des sciences, tome 121 (1895), p. 288.

M. Lé vy: Note sur les diverses manières d'appliquer la règle du trapèze au calcul de la stabilité des barrages en maçonnerie. Ann. des p. et ch. 1897 . 4 me trim. p. 5.

L. W. Atcherley and Prof. Karl Pearson: On some disregarded points in the stability of masonry dams. Abstract in Min. of proc. Inst. C. E. vol. CLXII, p. 456.

Prof. Karl Pearson: On the stability of masonry dams. Engg. vol. 80 (1905), p. 35, 171.

Max Am Ende: Notes on stresses in masonry dams. Engg. vol. 80 (1905), p. 751.

J. S. Wilson and W. Gore: Stresses in Dams. An experimental investigation by means of india rubber models. Engg. vol. 80 (1905), p. 134, and Min. of proc. Inst. C. E. session 1907-1908.

E. P. Hill: Stresses in Masonry Dams. Ebenda.

J. W. Ottley and A.W. Brightmore: Experimental investigations of the Stresses in Masonry Dams subjected to waterpressure. Ebenda. 
Die Arbeiten von Maurice Lévy müssen fortan als die wichtigste Grundlage für die Berechnung hoher Staumauern gelten. Eine im Jahre 1904 veröffentlichte, elementare Berechnung der Staumauern von Prof. Kressnik ${ }^{1}$ ) führt Lévy's Arbeiten zwar an, geht jedoch nicht darauf ein. Das gleiche gilt von der Berechnungsweise, welche Tudsbery und Brightmore in ihrem Buche vorführen. ${ }^{2}$ ) Lévy selbst begnügt sich damit, die Aufgabe ganz allgemein zu behandeln, ohne sich auf Anwendungen einzulassen. Auch die übrigen Forscher weisen zwar auf Mängel der älteren Berechnungsarten hin, schlagen aber keine neue vor.

Ich habe daher den von Lévy gewiesenen Weg weiter verfolgt, meine ursprüngliche Abhandlung umgearbeitet und die Lösung der Aufgabe gefunden, welche ich den Fachgenossen hiermit übergebe. Sie bildet einen Abschnitt aus meinen Vorlesungen an der Münchener Technischen Hochschule und ist das schlichte Endergebnis vieljähriger Arbeit. Sie erfüllt die neueren Anforderungen, ist also vollständiger, richtiger und überdies genauer als die frühere. Trotzdem ist sie einfacher und übersichtlicher.

Immerhin bleibt die Berechnung einer hohen Staumauer auch jetzt noch eine mühsame Arbeit. Sie lohnt sich aber, denn es gibt kein anderes Mittel, um das kleinstmögliche Profil eines solchen Bauwerkes herzustellen und dadurch an einer großen Anlage Hunderttausende in einwandfreier Weise zu ersparen.

Die Berechnung ist so eingehend entwickelt, daß der weniger geübte Leser dem Gedankengange leicht zu

1) P. Kressnik: Das kleinstmögliche Profil der Talsperremauern. Zeitschr. d. Osterr. Ing.- u. Arch.-Ver. 1904, S. 534.

$\Rightarrow$ J. H. T. Tudsbery and A. W. Brightmore: Waterworks Engineering. 3. ed. London 1905, p. 210, 214, 230. 
folgen vermag, und, wie ich glaube, so übersichtlich dargestellt, daß der Geübte mit Dingen, die ihm nebensächlich erscheinen, sich nicht aufzuhalten braucht.

Als Einheiten halten wir fest: Meter und Tonne.

Wir betrachten stets ein Stück Mauer zwischen zwei Querschnittebenen im Abstande Eins. 


\section{Praktische Vorbedingungen.}

a) Sicherung der Mauer gegen den Auftrieb des Wassers.

Damit die Mauer nicht gefährdet wird durch Wasser, welches unter hohem Druck zwischen die Schichten dringt, genügt es nicht, daß die Druckspannung am inneren Rande bei vollem Teiche nicht kleiner wird als Null.

Nimmt man das spezifische Gewicht des Wassers als Einheit, so ist die Stärke (Intensität) des Wasserdruckes in der Tiefe von $y$ Metern gleich $y$ t/qm.

Ist (in t/qm) $\beta_{m}$ die Stärke des Randdruckes im Gemäuer auf der Wasserseite in der Tiefe $y$, so hätte man an Stelle der Bedingung $\beta_{m}=0$ zu setzen.

$$
\beta_{m} \geqq y .
$$

Diese Bedingung bezieht sich auf wagerechte Lagerfugen; dann kann das Wasser nicht mehr durch den hydrostatischen Druck hineingepreßt werden, sondern es ist ein Bestreben vorhanden, es hinauszupressen. Will man aber an der Bedingung festhalten, daß die Spannung am inneren Rande der Lagerfläche bei vollem Teiche den Wert Null habe, so kann man die von Lévy vorgeschlagene Schutz- oder Schirmmauer an- 
Sicherung der Mauer gegen den Auftrieb des Wassers.

pringen, wie sie beim Réservoir du Ban ausgeführt wurde. (Abb. 1.)

An der Wasserseite der Staumauer $S$ stellt man lotrechte Rippen oder Pfeilervorlagen $P$ her, an welche die Schirmmauer $M$ sich anlehnt. Hierdurch bildet man Schächte von etwa $2 \mathrm{~m}$ im Geviert, die sich über die ganze innere Wandfläche der Staumauer erstrecken. Ein allenfalls entstehender Sprung führt das eindringende Wasser in einen oder mehrere von diesen Schächten, aus denen man es ableiten kann, ohne ihm zu gestatten, daß es eine bedenkliche Höhe erreicht. Aus der Wasserführung der Entwässerdohle oder -Röhre wird man gewahr, was hinter der Staumauer vorgeht. Ist die Abflußmenge beträchtlich, so kann man die Schächte untersuchen und die Sprünge verstopfen.

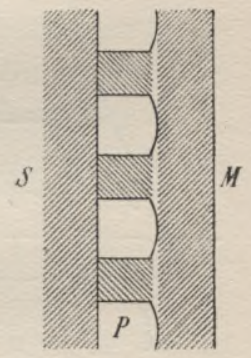

Abb. 1.

Gegen lotrechte Sprünge schützt bogenförmiger Grundriß. Der Wasserdruck auf das Gewölbe wird die Sprünge dichten, da der Druck in den Stoßfugen an sich größer sein wird als der Wasserdruck.

Auf die Bogenform wird bei der folgenden Berechnung nicht Rücksicht genommen. Wir betrachten also die Staumauern als freistehend, nicht als eingespannt.

Wichtig ist es, von Fall zu Fall das Gemäuer, welches $\mathrm{zu}$ einer Staumauer verwendet werden soll, in einem mechanisch-technischen Laboratorium prüfen zu lassen, um nicht nur seine Druckfestigkeit, sondern auch seine Scherfestigkeit sowie seine Ausdehnungs- und Elastizitätverhältnisse festzustellen. Geringere Bedeutung hat die Zugfestigkeit, da es sich vermeiden läßt und stets vermieden werden sollte, Gemäuer auf $\mathrm{Zug}$ zu beanspruchen. 


\section{b) Grundgestalt des Mauerquerschnittes.}

Der Wasserspiegel reiche bis an die Krone (Abb. 2). Diese Annahme entspricht einem ungünstigsten Falle. Wir gehen also dabei sicher. Es sei Eins die Schwere

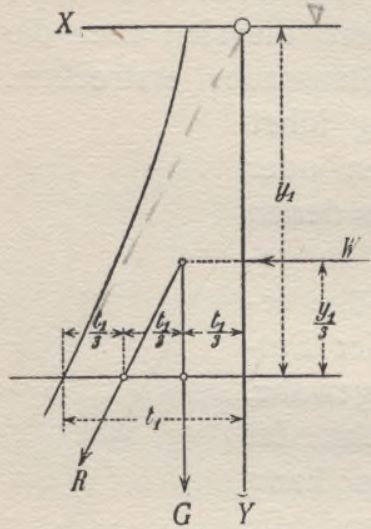

Abb. 2. des Wassers, $\gamma$ die des Gemäuers in $\mathrm{t} / \mathrm{cbm}$.

Wir stellen die Bedingung, bei vollem Teiche solle die Spannung am inneren Rande der wagerechten Lagerfugen Null werden, bei leerem Teiche am äußeren. Die eine Drucklinie hat daher am äußeren, die andere am inneren Kernrande zu verlaufen, somit hat jede der beiden Drucklinien durch ein Drittel der Fugenbreite zu gehen ${ }^{1}$ ).

Die innere Wand sei eine lotrechte Ebene. Für eine wagerechte Fuge in der Tiefe $y_{1}$ erhalten wir die Gleichgewichtbedingung wie folgt:

a) Die Schwerlinie soll durch das innere Drittel der wagerechten Fuge gehen, daher

$$
\begin{aligned}
\frac{1}{3} t_{1} \int_{0}^{y_{1}} t d y & =\int_{0}^{y_{1}} \frac{1}{2} t^{2} d y \\
t_{1} \int_{0}^{y_{1}} t d y & =\frac{3}{2} \int_{0}^{y_{1}} t^{2} d y
\end{aligned}
$$

b) Die Mittelkraft aus dem Wasserdruck und dem Gewichte der Mauer soll durch das äußere Drittel gehen:

$$
\frac{1}{6} y_{1}^{3}=\gamma \cdot \frac{1}{3} t_{1} \int_{0}^{y_{1}} t d y \text { oder } \frac{1}{2 \gamma} y_{1}{ }^{3}=t_{1} \int_{0}^{y_{1}} t d y,
$$

1) Diese Annahme setzt die Zulässigkeit der sog. „Trapezregel“ voraus. Wir kommen darauf wieder zurïck. 
das ist nach obigem,

differenziert:

$$
\frac{1}{2 \gamma} \cdot y_{1}^{3}=\frac{3}{2} \int_{0}^{y_{1}} t^{2} d y
$$

$$
\begin{aligned}
\frac{3}{2} \cdot \frac{1}{\gamma} \cdot y_{1}^{2} & =\frac{3}{2} t^{2} \\
\frac{y}{t} & =\sqrt{\gamma}
\end{aligned}
$$

Der Querschnitt ist daher ein rechtwinkeliges Dreieck. Die innere Kathete ist lotrecht. Die Breite $t$ in irgendeiner Tiefe $y$ ist nach Gl. (1) zu berechnen.

Beide Stützlinien sind Gerade und begrenzen das mittlere Drittel der Querschnittfläche.

Der Randdruck wächst, wie sich leicht zeigen läßt, proportional mit der Höhe $y$.

In der Ausführung kann man die Mauer oben nicht in einer scharfen Kante enden lassen, sondern die Krone $\mathrm{muß}$ mindestens $\mathrm{zu}$ einer Brüstung ausgebildet werden. Häufig wird über die Mauerkrone ein Weg geführt, am einfachsten, indem man den oberen Teil der Mauer zu einem rechtkantigen Block ergänzt. Allein, auch wenn die Fahrbahn auf irgendeinem besonderen Unterbau - einer Art Jochbrücke aus Holz oder Eisen, oder Bogenstellungen - ruht, muß man dies Bauwerk, für den Zweck der Berechnung, durch einen statisch gleichwertigen Mauerklotz ersetzt sich denken.

Wenn man aber die dreikantige Grundform durch einen solchen Aufbau oben belastet, so werden die Stützlinien durchweg verschoben; und wenn man alsdann noch die Bedingung gleicher Standsicherheit beibehalten will, so wird sich für den, unterhalb des rechtkantigen Aufbaues oder "Kopfes" gelegenen Teil des Mauerquerschnittes eine Form ergeben, welche um so mehr von der dreikantigen Grundform abweicht, je 
breiter die Krone, kurz, je größer und schwerer jener wirkliche oder stellvertretende Mauerklotz ist.

c) Lagerflächen freistehender Staumauern.

In einem prismatischen Körper, wie eine Staumauer, gibt es in jedem Querschnitte zwei Gruppen von Linien, die sich überall rechtwinklig schneiden und welche Lamé die isostatischen Linien genannt hat. Sie haben die Eigenschaften:

a) $\mathrm{Da} ß$ sie nur streng senkrechte (normale) Drücke erfahren,

b) daß in jedem Punkte die eine Linie die größte, die andere die kleinste Druckspannung erleidet, welche an diesem Punkte entstehen.

Die Linien der ersten Gruppe sollen $A$, die der zweiten $B$ heißen.

Offenbar wären nach der Liniengruppe $A$ die Lagerfugen anzulegen, da hierdurch jedes Bestreben, längs der Lagerflächen zu gleiten, vermieden würde. Die Erfüllung dieser Bedingung vereinfacht sich, da man vorweg zwei isostatische Linien kennt, d. h. Linien, die nur normale Drücke erleiden. Das sind die beiden Häupter des Staumauerquerschnittes.

Das äußere oder talseitige Haupt entspricht notwendigerweise der Gruppe $B$, wenn es den Druck Null erleidet. Das innere, berg- oder wasserseitige Haupt gehört ebenfalls zu dieser Gruppe, wenn die Bedingung

erfüllt ist.

$$
\beta_{m} \geqq y
$$

Folglich müssen alle Linien $\operatorname{der}$ Gruppe $A$, d. i. alle Lagerfugen, die beiden Stirnen rechtwinkelig schneiden.

Es genügt, die Lagerfugenlinien durch Kreisbögen zu ersetzen, welche die Stirnen rechtwinkelig schneiden. 
Dann wird an den Stirnen kein Bestreben, zu gleiten, vorhanden sein und im Inneren nur ein schwaches, was sehr wichtig ist, namentlich für die Grundfläche des Dammes. Das hindert natürlich nicht, unregelmäßiges Bruchsteingemäuer und Stufen an der Grundfläche anzuwenden. Diese Art zu mauern, erleichtert auch den Abfluß der Niederschläge während der Arbeit, namentlich, wenn sie zeitweise unterbrochen werden muß. 


\section{Theoretische Vorbedingungen.}

a) Praktische Zulässigkeit der Trapezregel.

Gröfse der Randspannungen.

Die Trapezregel oder die Annahme, daß die Belastungsfläche über der Angriffachse ein Trapez sei, beruht bekanntlich auf Naviers Hypothese vom ebenen Querschnitt und dem Satze vom festwertigen Elastizitätmaß. Sie wurde bei der Berechnung von Staumauern angewendet

1. durch Delocre auf die wagerechten Schnitte,

2. durch Bouvier auf die Schnitte normal zur Druckkurve,

3. durch Guillemain, ausgehend von demjenigen Schnitte durch jeden Punkt der talseitigen (äußeren) Stirne, welcher den größten Normaldruck in diesem Punkte ergibt.

Da der durch Guillemain vorgeschlagene Weg den stärksten Druck ergäbe, so würde die Vorsicht empfehlen, ihn einzuschlagen, obschon er der mühsamste wäre.

Lévy weist nach, daß praktisch die Berechnungsarten von Delocre, Bouvier und Guillemain annähernd gleichwertig sind; daß die Trapezregel genau ist, $d . h$. den Grundsätzen der mathematischen Elastizitätstheorie entspricht für dreieckige Querschnitte, d. i. mit der 
Kronenbreite Null, nicht aber für trapezförmige Querschnitte und noch weniger für Staumauern mit nicht ebenen Stirnen. Allein, da die Trapezregel für dreieckige Profile richtig ist, so darf man annehmen, daß sie noch annähernd richtig sein werde für Profile, die vom Grunddreieck nicht allzusehr abweichen.

Was die Größe der Randspannungen betrifft, so wird tatsächlich der stärkste Druck ausgeübt auf das Element, welches normal ist zur äußeren (talseitigen) Stirne. Das ist streng richtig, d. h. unabhängig von der Trapezregel wie von jeder anderen Hypothese.

Es sei $a b$ (Abb. 3) ein Flächenelement (Länge $1 \mathrm{~m}$ ), normal zur Stirne im Punkte $a$.

ac sei ein beliebiges Element, mit der Normalen einen Winkel $\vartheta$

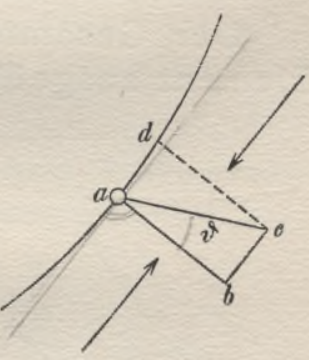

Abb. 3. einschließend. $c b$ sei gleichlaufend zur Berührenden im Punkte $a$.

Das unendlich kleine Prisma $a b c$ ist im Gleichgewichte unter dem Einflusse erstens der Drücke, die auf seine Seitenflächen wirken, zweitens seines Gewichtes.

Die Drücke sind unendlich klein von erster Ordnung, das Gewicht ist unendlich klein von zweiter Ordnung und kann vernachlässigt werden.

Dasselbe gilt von dem Drucke auf das mit der Wandfläche gleichlaufende Element $b c$; denn, wenn wir das Rechteck abcd vervollständigen, so erleidet das Element ad auf der Wand einen Druck Null, daher ist die Stärke des Druckes auf das Element $b c \| a d$ unendlich klein, und diese, multipliziert mit dem Flächeninhalt, ist unendlich klein von zweiter Ordnung. 
Das Dreieck $a b c$ ist somit vollkommen im Gleichgewichte unter dem Einflusse bloß der zwei Drücke, welche auf die Seiten $a b$ und $a c$ wirken. Diese Drücke müssen daher gleich und entgegengesetzt sein und längs einer Geraden wirken, welche die Mitten der beiden Seiten verbindet, $d . h$. sie sind gleichlaufend mit der Berührenden an der Stirne im Punkte $a$.

Daher der Satz:

Der Gesamtdruck, welcher auf irgendein Element ausge übt wird, das von einem Punkte der talseitigen (äußeren)

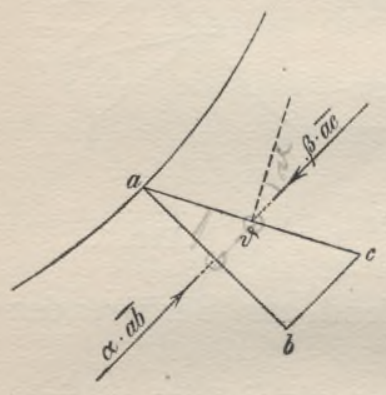

Abb. 4. Böschung ausgeht, ist parallel zur Böschung in diesem Punkte; woraus derFolgesatz:"

Der Druck auf ein zur talseitigen Böschung senkrechtes Element ist senkrecht zu diesem Elemente.

Es sei (Abb. 4) $\alpha$ die Stärke des Druckes auf das Element $a b$, welches in $a$ auf der Böschung senkrecht steht; $\beta$ die Stärke des Druckes auf das Element $a c$, welches mit dem senkrechten Elemente den Winkel $\vartheta$ macht. Dann sind die auf die Elemente ausgeübten Drücke $\alpha \cdot \overline{a b}$ und $\beta \cdot \overline{a c}$, und, da sie im Gleichgewichte sind,

wonach

$$
\begin{gathered}
\alpha \cdot \overline{a b}=\beta \cdot \overline{a c} \\
\beta=\frac{\overline{a b}}{\overline{a c}} \cdot \alpha=\alpha \cdot \cos \vartheta
\end{gathered}
$$

Sind $\beta_{n}$ und $\beta_{t}$ die normale und die tangentiale Komponente von $\beta$, so wird man haben

$$
\beta_{n}=\beta \cos \vartheta=\alpha \cos ^{2} \vartheta \text {. . . . }
$$




$$
\beta_{t}=\beta \sin \vartheta=\alpha \cos \vartheta \sin \vartheta=\frac{\alpha}{2} \sin 2 \vartheta .
$$

Nach dem ersten Ausdrucke wird $\beta_{n}$ ein Größtes für $\vartheta=0$ und wird gleich $\alpha$, d. h. das normale Element erleidet den stärksten Druck. Wenn man daher irgendwie die senkrechte Druckstärke $\beta_{n}$ gefunden hat, die auf ein Element ausgeübt wird, welches den Winkel $\vartheta$ mit dem normalen Elemente macht, so ist der größte Druck auf die Flächeneinheit des normalen Elementes

$$
\alpha=\frac{\beta_{n}}{\cos ^{2} \vartheta} \text {. }
$$

Bemerkt man noch, daß nach Formel (3) die größte Scherkraft für ein Element der talseitigen (äußeren) Böschung stattfindet bei

$$
\vartheta=45^{0}
$$

und daß sie immer gleich ist $\frac{\alpha}{2}$, so folgt der für die Zerstörung von Staumauern wichtige Satz: Die größte Scherspannung an einem Punkte der äußeren Böschung tritt auf in den zwei unter $45^{0}$ gegen diese Böschung geneigten Richtungen und erreicht immer die Hälfte der größten Druckstärke $\alpha$.

b. Festsetzung der praktischen Grenze für die Beanspruchung des Gemäuers.

Ist $a c$ wagerecht (Abb. 5), so wird die Richtung von $\beta_{n}$ lotrecht, der Winkel $\vartheta$ wird zum Böschungswinkel $\vartheta_{0}$ im Punkte $a$ und es ist

$$
\cos ^{2} \vartheta_{0}=\left(\frac{d y}{d s}\right)^{2}=\frac{d y^{2}}{d y^{2}+d t^{2}}=\frac{1}{1+\left(\frac{d t}{d y}\right)^{2}}=\frac{1}{1+\varepsilon^{2}} \ldots
$$

wo $\varepsilon$ das Böschungsverhältnis im Punkte $a$ bezeichnet. Hätte man nach der Trapezregel den lot- 
rechten Randdruck $\beta_{n}$ im Punkte $a$ gefunden (Abb. 6), so wäre [Gl. (2)]

$$
\alpha=\frac{\beta_{n}}{\cos ^{2} \vartheta_{0}}=\left(1+\varepsilon^{2}\right) \beta_{n} \quad . \quad . \quad .
$$

und die zu berücksichtigende größte Scherspannung im Punkte $a$

$$
\sigma=\frac{\alpha}{2}=\frac{1+\varepsilon^{2}}{2} \beta_{n} . . . \quad .
$$

oder, wenn $\sigma_{0}$ die zulässige Scherspannung ist, wie man sie für die gegebene Stampfmörtel- oder Mauer-

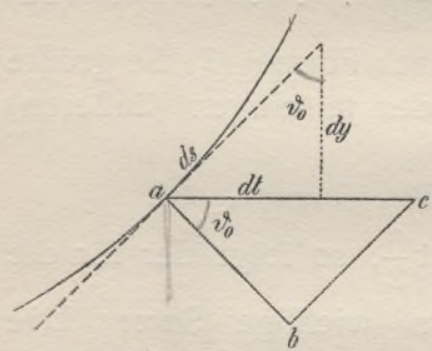

Abb. 5.

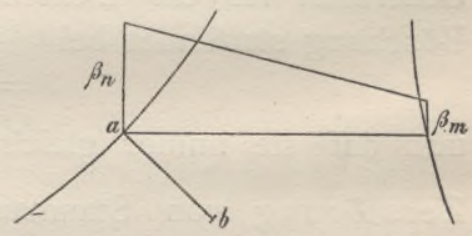

Abb. 6.

werkgattung auf Grund von Festigkeitversuchen festgesetzt hat, so darf $\beta_{n}$ nicht größer sein als

$$
\max \beta_{n} \leqq \frac{2 \sigma_{0}}{1+\varepsilon^{2}} .
$$

Versuche zur Bestimmung der Scherfestigkeit des $\mathrm{zu}$ einer freistehenden Staumauer $\mathrm{zu}$ verwendenden Betons oder Gemäuers sind also mindestens ebenso wichtig, wie die bloßen Zerdrückversuche.

Brik fand die Scherfestigkeit von Stampfbeton (1 Portlandzement, 2 Sand, 2 Schotter)

$$
\sigma=34,5 \mathrm{~kg} / \mathrm{qcm}=345 \mathrm{t} / \mathrm{qm} \text {. }
$$

Nach Wittmann's Versuchen ist die Scherfestigkeit von Beton gleich $1 / 7$ bis $1 / 10$ seiner Druckfestigkeit $\delta$. 
Praktische Grenze der Beanspruchung.

Bach fand für feinkörnigen, blauen Granit

$\delta=1006 \mathrm{~kg} / \mathrm{qcm}, \quad \sigma=45 \mathrm{~kg} / \mathrm{qcm}, \quad \frac{\sigma}{\delta}=\frac{1}{22}$.

Nach Bauschinger ist im Durchschnitt

für Stein $\sigma=1 / 13 \delta$,

für Zementmörtel $\sigma=1 / 6 \delta$.

Es scheint, daß Beton eine verhältnismäßig größere Scherfestigkeit besitzt, als Mauerwerk. Die Druckfestigkeit von Zementbeton ist aber im Durchschnitt geringer als die von Steinen.

Föppl hat beobachtet, daß Beton gegen Stöße viel weniger widerstandfähig ist als Sandstein von der gleichen Druckfestigkeit.

Man sollte also von Fall zu Fall möglichst gründliche Versuche anstellen lassen oder sich genau derjenigen Mischungen bedienen, für

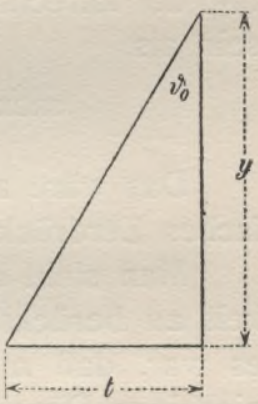

Abb. 7. welche bereits die Ergebnisse von Versuchen an großen Probekörpern vorliegen.

Es bezeichne $\gamma$ das spezifische Gewicht des Gemäuers, so hat man für das Grunddreieck (Abb. 7) nach Gl. (1)

$$
\frac{t}{y}=\tan \vartheta_{0}=\frac{1}{\sqrt{\gamma}}=\varepsilon .
$$

$\gamma$ liegt zwischen den Werten 2 und 3, und im Durchschnitt kann man annehmen $\gamma=2,3$.

Ist daher $\beta_{n}$ die in der gewöhnlichen Weise gefundene Stärke des lotrechten Randdruckes, dann ist bei obigem Grunddreieck die Stärke des Randdruckes längs der Böschung

$$
\alpha=\frac{\beta_{n}}{\cos ^{2} \vartheta_{0}}=\left(1+\varepsilon^{2}\right) \beta_{n}=\frac{\gamma+1}{\gamma} \beta_{n} .
$$


und die Stärke der Scherkraft daselbst

$$
\begin{gathered}
\sigma=\frac{\alpha}{2}=\frac{\gamma+1}{\gamma} \cdot \frac{\beta_{n}}{2} \\
\gamma=2,3, \quad \sigma=0,72 \beta_{n} .
\end{gathered}
$$

also für

Verwendet man denselben Stampfmörtel wie Brik, so hat man bei fünffacher Sicherheit

$$
\sigma_{0}=\frac{345}{5}=69,0 \mathrm{t} / \mathrm{qm},
$$

und der lotrecht gemessene Randdruck darf nicht größer sein als

$$
\max \beta_{n}=\frac{69}{0,72}=96 \sim 100 \mathrm{t} / \mathrm{qm} \text {. }
$$

Das wäre aber etwa 30 fache Sicherheit gegen einfaches Zerdrücken.

Man sieht also, wie wichtig es ist, die Scherfestigkeit $\mathrm{zu}$ berücksichtigen, und welch glücklicher Zufall es war, daß man bei den älteren Staumauern an so niedrigen Druckbeanspruchungen festgehalten hat.

Da ferner beim Grunddreieck

$$
\beta_{n}=\frac{2 \cdot \frac{t \cdot y}{2} \cdot \gamma}{t}=\gamma \cdot y
$$

so wird nach $(5 \mathrm{~b})$

$$
\sigma=(\gamma+1) \frac{y}{2} .
$$

Die Schubkraft auf einer wagerechten Ebene in der Tiefe $y$ ist gleich dem Wasserdruck auf die lotrechte Wand und beträgt in Tonnen

$$
S_{y}=\frac{y^{2}}{2} \text {. }
$$

Ihre mittlere Stärke ist $\sigma_{y}=\frac{S_{y}}{t}$ und da $t=\frac{y}{\sqrt{\gamma}}$

$$
\sigma_{y}=\frac{S_{y}}{t}=\frac{y^{2}}{2 y} \sqrt{\gamma}=\frac{y}{2} \sqrt{\gamma} .
$$


Nach den Modellversuchen von Wilson und Gore ${ }^{1}$ ) kann man annehmen, daß diese Schubspannung sich über die wagerechte Ebene nach dem Geradliniengesetze verteilt, wie die Normalspannung, so daß sie am Rande der Böschung den Größtwert erreicht:

$$
\max \sigma_{y}=y \sqrt{\gamma}
$$

Da $\gamma$ zwischen 2 und 3 liegt, so hat man

und

$$
\text { nach (5c) } \quad \sigma=1,5 \text { bis } 2
$$

nach $(5 \mathrm{e}) \max \sigma_{y}=1,4,1,7$.

Man geht also sicher, wenn man mit der Scherspannung in der Böschungsebene rechnet, da sie am größten ist und ein $\mathrm{Ab}-$ scheren längs einer wagrechten Ebene ist leicht $\mathrm{zu}$ vermeiden, wenn die Böschungsebene widersteht.

Schubkräfte auf lotrechten Ebenen. Auf der Grundfläche $a b$ (Abb. 8) verteilt sich das Gewicht nach dem Dreieck $a b d$ und der nach aufwärts gerichtete

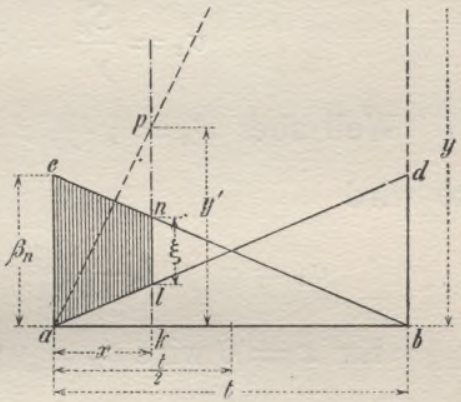

Abb. 8. Gegendruck nach dem Dreieck $a b c$. Betrachten wir die lotrechte Ebene $k p$.

Die links von $k p$ wirkenden Kräfte sind: das Gewicht von $k p a$, dargestellt durch das Dreieck $a k l$ und der Gegendruck, dargestellt durch das Trapez $a k n c$. Beide Kräfte sind parallel, daher ist ihr Unterschied die gesamte Schubkraft auf $k p$, dargestellt durch die Fläche des Trapezes $a l n c$.

1) Eine strenge mathematische Untersuchung hat Professor O. M o h r veröffentlicht: Der Spannungszustand einer Staumauer. Zeitschr. d. Österr. Ing.- u. Arch.-Ver. 1908, Nr. 40 u. 41. 
Mit den Bezeichnungen der Abbildung erhalten wir:

$$
\frac{\xi}{\beta_{n}}=\frac{\frac{t}{2}-x}{\frac{t}{2}}, \xi=\left(1-\frac{2 x}{t}\right) \beta_{n}
$$

und die gesamte Schubkraft

$$
S_{x}=\left(\beta_{n}+\xi\right) \frac{x}{2}=\beta_{n}\left(1-\frac{x}{t}\right) x .
$$

Nach den erwähnten Versuchen von Wilson und Gore kann angenommen werden, daß diese Schubkraft über die lotrechte Ebene sich gleichmäßig verteilt; ihre Stärke ist daher gleich ihrem mittleren Werte

$$
\sigma_{x}=\frac{S_{n}}{y^{\prime}}=\beta_{n}\left(1-\frac{x}{t}\right) \frac{x}{y^{\prime}} .
$$

Weil aber $\beta_{n}=\gamma \cdot y, t=\frac{y}{\sqrt{\gamma}}, y^{\prime}=x \sqrt{\gamma}$,

so wird

$$
\sigma_{x}=\gamma \cdot y\left(1-\frac{x \sqrt{\gamma}}{y}\right) \frac{1}{\sqrt{\gamma}}=y \gamma \bar{\gamma}\left(1-\frac{x \sqrt{\gamma}}{y}\right)
$$

für $x=0$ wird $\sigma_{x}=y \sqrt{\gamma}$, wie (5e)

$$
\begin{array}{rlrl}
\# x=\frac{t}{2} \quad \# \quad \sigma_{x}=\frac{y}{2} \sqrt{\gamma}, \quad, & \\
, x=t \quad \# \quad \sigma_{x}=0 .
\end{array}
$$

Dies ist das nämliche Geradliniengesetz, nach welchem auch die Stärke der Schubspannung auf der Grundebene $a b$ sich ändert.

Längs eines Lotes sind die Stärken der Schubspannungen auf den wagerechten und auf den lotrechten Flächenelementen durchweg gleich und festwertig.

Dies alles gilt zunächst für das Grunddreieck, ist aber noch anzunehmen für Querschnittformen, die vom Grunddreieck wenig abweichen. 


\section{Entwickelung der Bestimmungsgleichungen.}

a) Hypothesen und Bezeichnungen.

Das spezifische Gewicht des Gemäuers, zugleich die Schwere in $t / c b m$, ist, wie bereits erwähnt, 2 bis 3 .

Die Punkte des Querschnittes seien auf zwei rechtwinkelige Koordinatenachsen bezogen (Ab.9). Die eine, $O X$, wagerecht längs der Krone der Staumauer von der Bergseite gegen die Talseite, die andere, $O Y$, von der oberen Kronenkante lotrecht nach abwärts, also längs der wasser- (oder berg-) seitigen Stirne verlaufend, wenn diese lotrecht ist, was wir vorläufig annehmen.

In der Tiefe $y$ sei die Dicke der Mauer, wagerecht gemessen,

$$
t=f(y) \text {. . . }
$$

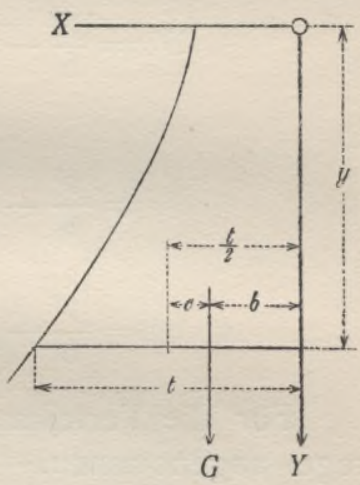

Abb. 9.

Der Druck des Wassers auf die Wandfläche von der Höhe $y$ und der Breite Eins ist, da ja das spezifische Gewicht des Wassers oder dessen Schwere, in t/cbm, gleich Eins $\frac{y^{2}}{2}$. 
III. Entwickelung der Bestimmungsgleichungen.

Das Gewicht des der Höhe $y$ entsprechenden Gemäuers ist

$$
G=\gamma \int_{0}^{y} t d y \quad . \quad . \quad . \quad . \quad .
$$

Biegendes Moment gleich Moment des Wasserdruckes weniger Moment des Gewichtes in bezug auf die Mitte

$$
\begin{gathered}
\mathfrak{2}=\frac{y^{3}}{6}-G\left(\frac{t}{2}-b\right) \\
b=\frac{\int_{0}^{y} \frac{t^{2}}{2} d y}{\int_{0}^{y} t d y}, \\
2 \pi=\frac{y^{3}}{6}-\gamma \int_{0}^{y} t d y\left(\frac{t}{2}-\frac{\int_{0}^{y} \frac{t^{2}}{2} d y}{\int_{0}^{y} t d y}\right) \\
=\frac{y^{3}}{6}-\frac{\gamma}{2}\left(t \int_{0}^{y} t d y-\int_{0}^{y} t^{2} d y\right) \\
=\frac{y^{3}}{6}+\frac{\gamma}{2}\left(\int_{0}^{y} t^{2} d y-t \int_{0}^{y} t d y\right) .
\end{gathered}
$$

folglich

Für die Verteilung des Druckes wird die Trapezregel angenommen.

b) Widerstand gegen das Gleiten längs einer wagerechten Schnittebene.

Kleinstes Gewicht des Gemäuers.

Ist $\mu$ die Reibungsziffer, so muß sein:

$$
G \geqq \frac{y^{2}}{2 u}
$$


oder die kleinste Querschnittfläche

$$
\int_{0}^{y} t d y \geqq \frac{y^{2}}{2 \mu \gamma} \text {. . . . . . }
$$

Es ist $\mu=0,7$ bis 0,75 , zwar etwas hoch für wagerechte Schichten, nicht aber für bogenförmige, bei denen für die wagerechte Schnittebene auch noch die Scherfestigkeit des Gemäuers in Betracht kommt.

Der kleinste Wert von $G$ ist, für die Höhe $y$,

$$
G=\frac{y^{2}}{2 \mu}
$$

und für das ganze Bauwerk von der Höhe $h$ wäre er $\frac{h^{2}}{2 \mu}$. Differenziert man die Gleichung, so erhält man, $\operatorname{da} G=\gamma \int_{0}^{y} t d y, \quad t=\frac{y}{\mu \gamma}$,

so daß man für den geringsten Aufwand an Gemäuer einen dreieckigen Querschnitt anzuwenden hätte. Die äußere Wand hat einen Anzug

$$
1: \mu \gamma
$$

und wenn $\operatorname{man} \mu=0,7, \gamma$ zwischen 2 und 3 annimmt, so wäre der Anzug

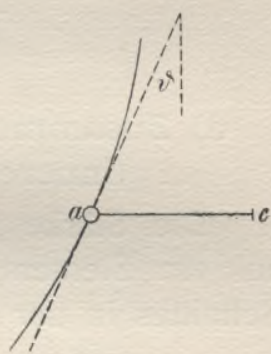

Abb. 10.

$$
1 \cdot 1,4 \text { bis } 1: 2,1 \text {. }
$$

c) Zusammendrückung an der talseitigen (äufseren) Böschung. (Abb. 10.)

Hier gilt der Satz:

Der größte Druck an einem Punkte $a$ der äußeren Stirne ist gleich dem Druck, der auf dem wagerechten Element entsteht, das durch den Punkt a geht, geteilt durch das Quadrat des Cosinus von dem Winkel, den die Böschung 
mit dem Lote macht. Durch Gleichung (2b), Seite 18, ist diese Beziehung gegeben.

Der Druck $\alpha=\left(1+\varepsilon^{2}\right) \beta_{n}$ darf die zulässige Beanspruchung des Gemäuers nicht überschreiten, und zwar sollte er mit Rücksicht auf die Scherfestigkeit bemessen werden nach Gl. (3a) und (5). Die Trapezregel ergibt

$$
\beta_{n}=\frac{G}{t}+\frac{62 \pi}{t^{2}},
$$

wonach

$$
\frac{2 \sigma_{0}}{1+\varepsilon^{2}} \cdot t^{2} \geqq G t+6 \mathfrak{m} \text {. . . . }
$$

$G$ und $2 \mathfrak{r}$ sind aus Gl. (7) und (8) zu nehmen. Die Bedingung gleichen Widerstandes längs der talseitigen (äußeren) Böschung lautet daher

$$
\frac{2 \sigma_{0}}{1+\varepsilon^{2}} \cdot t^{2}=G t+62 \pi \text {. . . . }
$$

d) Zusammendrückung an der bergseitigen (inneren) Böschung (vgl. Abb. 6).

Die Stärke $\beta_{m}$ des lotrecht gemessenen Druckes auf ein Element am inneren Rande des wagerechten Schnittes in der Tiefe $y$ ist

$$
\beta_{m}=\frac{G}{t}-\frac{62 \pi}{t^{2}} .
$$

Die Größen $t, G, 2 \pi$ sind aus den vorangegangenen Berechnungen bekannt.

Wenn man eine Schutzmauer hat und den Frost nicht zu fürchten braucht, so sollte man sich versichern, daß überall

oder

$$
\begin{gathered}
\beta_{m} \geqq 0 \\
G t-62 \mathfrak{N} \geqq 0 \\
\text { esetzten Falle müßte } \\
\beta_{m} \geqq y \\
G t-62 \mathfrak{N} \geqq t^{2} y .
\end{gathered} .
$$

Im entgegengesetzten Falle müßte man sicher sein, daß überall

oder 
Grenzhöhe oder praktische Grenze des Grunddreieckes. 27

Diese Bedingung wird wahrscheinlich nicht von selber erfüllt sein und eine neue Verstärkung der Mauer verlangen.

e) Grenzhöhe oder praktische Grenze für die Gültigkeit des Grunddreieckes.

Die Grenzhöhe ist erreicht, wenn das Gewicht des Dreikantes $\frac{h t}{2}$, das auf die Grundfläche $t$ sich nach einem Dreieck verteilt (Abb. 11) dort einen Druck erzeugt, dessen mittlere Stärke halb so groß ist wie die zulässige Beanspruchung $\beta$. Wir haben also für die äußere oder untere Stirne

$$
h_{u} \cdot t_{u} \cdot \frac{\gamma}{2}=t_{u} \cdot \frac{\beta_{n}}{2} ; h_{u}=\frac{\beta_{n}}{\gamma}
$$

und für die innere oder obere Stirne

$$
h_{0}=\frac{\beta_{m}}{\gamma} \text {. }
$$

Da für die obere, lotrechte Stirne $\vartheta_{0}=0$, also $\varepsilon=0$, so ist, wenn wir Gl. (5) benutzen

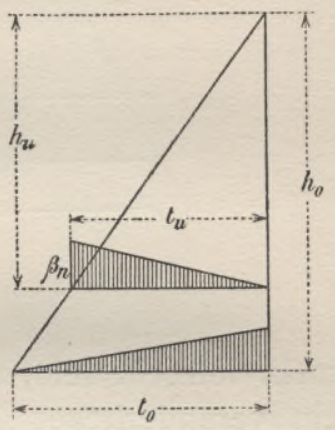

Abb. 11.

$$
\max \beta_{n}=\frac{2 \sigma_{0}}{1+\varepsilon^{2}}, \quad \max \beta_{m}=2 \sigma_{0}
$$

und, da ferner für das Grunddreieck $\tan \vartheta_{0}=\frac{t}{h}=\frac{1}{\sqrt{\gamma}}$ $=\varepsilon$, so ergibt sich

$$
\begin{gathered}
h_{u}=\frac{2 \sigma_{0}}{\left(1+\varepsilon^{2}\right) \gamma}=\frac{2 \sigma_{0}}{\left(1+\frac{1}{\gamma}\right) \gamma}=\frac{2 \sigma_{0}}{\gamma+1} . \\
h_{0}=\frac{2 \sigma_{0}}{\gamma}, \frac{h_{u}}{h_{0}}=\frac{\gamma}{\gamma+1} . . .
\end{gathered}
$$


f) Fall, wo die bergseitige (innere) Wand nicht lotrecht ist. Notwendigkeit einer inneren Böschung.

Da die Randspannung an der lotrechten Innenwand proportional mit der Tiefe $y$ wächst bis die zulässige Grenze der Belastung, somit die Grenze für die Höhe einer lotrechten Wand erreicht ist, so kann für Höhen größer als $h_{0}$ die innere Wand von $y=h_{0}$ ab nicht

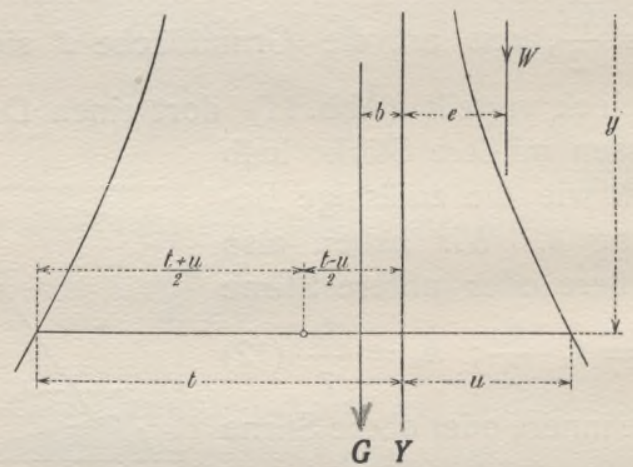

Abb. 12.

mehr lotrecht sein, sondern muß eine Böschung, sogar ein veränderliches Böschungsverhältnis erhalten. Die vorangegangenen Formeln sollen daher nach dieser Richtung vervollständigt werden. Es seien, in der Tiefe $y$ (Abb. 12), die Abszissen eines Profilpunktes außen $t$, innen $u$, so daß $t$ und $u$ zwei Funktionen von $y$ sind.

Der normale Druck auf den wagerechten Schnitt in der Tiefe $y$ ist ausgedrückt durch

$$
P=G+W
$$

wo $W$ die lotrechte Komponente des Wasserdruckes auf die wasserseitige Böschung bezeichnet. Es ist also

$$
P=\gamma \int_{0}^{y}(t+u) d y+\int_{0}^{y} y d u \text {. . . }
$$


Fall, wo die bergseitige Wand nicht lotrecht ist.

Das biegende Moment in diesem Schnitte ist

$$
\mathfrak{2}=\frac{y^{3}}{6}-G\left(\frac{t-u}{2}-b\right)-W\left(e+\frac{t-u}{2}\right) .
$$

Wir haben

$$
\begin{gathered}
G=\gamma \int_{0}^{y}(t+u) d y \\
b=\frac{\int_{0}^{y} \frac{t-u}{2}(t+u) d y}{\int_{0}^{y}(t+u) d y}=\frac{\int_{0}^{y} \frac{t^{2}-u^{2}}{2} d y}{\int_{0}^{y}(t+u) d y} \\
W=\int_{0}^{y} y d u, e=\frac{\int_{0}^{y} u y d u}{\int_{0}^{y} y d u} .
\end{gathered}
$$

Daher

$$
\begin{aligned}
2=\frac{y^{3}}{6}+\frac{\gamma}{2} & \left\{\int_{0}^{y}\left(t^{2}-u^{2}\right) d y-(t-u) \int_{0}^{y}(t+u) d y\right\}- \\
& -\int_{0}^{y} u y d u-\frac{t-u}{2} \int_{0}^{y} y d u \ldots . .
\end{aligned}
$$

Die Stärke des Druckes am bergseitigen Rande wäre gegeben durch

$$
\min \beta_{m}=\frac{P}{t+u}-\frac{62 \pi}{(t+u)^{2}} . . .
$$

und die Stärke des talseitigen Randdruckes

$$
\max \beta_{n}=\frac{P}{t+u}+\frac{62 \pi}{(t+u)^{2}} . . .
$$

Die Gleichungen (13) und (14) gelten für den Fall, daß der Wasserspiegel bis an die Mauerkrone reicht. Bis hierher sind wir Lévy im wesentlichen gefolgt und gehen nun auf dem gefundenen Wege weiter. 
III. Entwickelung der Bestimmungsgleichungen.

Wenn der Teich leer ist, so erhalten wir die Belastung des wagerechten Schnittes in der Tiefe $y$

$$
G=\gamma \int_{0}^{y}(t+u) d y \text {. . . . . }
$$

und das biegende Moment

$$
\mathfrak{U}=\frac{\gamma}{2}\left[(t-u) \int_{0}^{y}(t+u) d y-\int_{0}^{y}\left(t^{2}-u^{2}\right) d y\right] .
$$

Die Stärke des Druckes wäre am bergseitigen Rande

$$
\max \beta_{m}=\frac{G}{t+u}+\frac{6 \mathfrak{L} \tau}{(t+u)^{2}} . . .
$$

und am talseitigen Rande

$$
\min \beta_{n}=\frac{G}{t+u}-\frac{6 \mathfrak{L}}{(t+u)^{2}}
$$

Man kann die Gleichungen (16) und (19) auch schreiben:

$$
\begin{array}{ll}
(t+u)^{2} \cdot \max \beta_{n}=(t+u) P+62 \mathfrak{r} & \cdot(16 \mathrm{a}) \\
(t+u)^{2} \cdot \max \beta_{m}=(t+u) G+62 \mathcal{2} & .(19 a)
\end{array}
$$

wobei die Bedingung (5) im Auge zu behalten ist, nämlich $\beta_{n} \leqq \frac{2 \sigma_{0}}{1+\varepsilon^{2}}$, was auch für $\beta_{m}$ gilt. 


\section{Anwendung.}

\section{A. Abteilung der Querschnittfläche.}

Wenn wir auf die dreikantige Grundform einen rechtkantigen Kopf aufsetzen, so kann dieser nur der Bedingung entsprechend hergestellt werden, da $\beta$ an seiner Grundfläche der äußere (talseitige) Druckmittelpunkt (bei vollem Teiche) an den Kernrand, also ins äußere Drittel rückt. Der innere Druckmittelpunkt (bei leerem Teiche) wird immer in die Mitte fallen. Im darunter liegenden Teile des Mauerquerschnittes, dem Rumpf, sollen die beiden Druckmittelpunkte durchweg an den entgegengesetzten Kernrändern liegen, d. h. die Stützlinien müssen mit den Kerngrenzen zusammenfallen. Wir haben also zwischen "Kopf" und "Rumpf “ noch ein Zwischenglied, einen „Hals“, einzuschalten, in welchem der Obergang stattfindet. ${ }^{1}$ )

Diesen Hals machen wir trapezförmig, auf der Wasserseite lotrecht, und geben ihm solche Abmessungen, da $\beta$ er sich oben an den Kopf anschließt und daß an seiner unteren Begrenzung die beiden Druckmittelpunkte

1) Die in meiner ersten Schrift eingeführten Bezeichnungen Kopf, Hals, Rumpf, Fub, behalte ich bei, wie überhaupt die vorstehenden Erklärungen, des Zusammenhanges halber, von dort zum Teil herübergenommen sind. 
je an die Ränder des mittleren Drittels fallen, wie es in dem darauffolgenden Rumpfe durchweg sattfinden soll.

Im Rumpfe wächst der Randdruck stetig, bis die Grenzhöhe erreicht ist, von welcher ab die Randspannung nicht mehr wachsen, sondern festwertig bleiben soll. Von hier ab können die Stützlinien nicht mehr den Grenzen der Kernfläche folgen, sondern sie müssen sich gegen die Mitte hereinziehen.

Diesen unteren Teil, den Fuß, könnte man theoretisch unbegrenzt hoch machen. Allein, da die Trapezregel, wie hervorgehoben, um so weniger zutrifft, je mehr die Querschnittform vom Grunddreieck abweicht, so werden wir auf eine praktische Grenze für die Höhe von Staumauern geführt, wie später gezeigt werden soll.

Schon Delocre hat in seiner Querschnittform drei durch wagerechte Gerade getrennte Abschnitte unterschieden: einen beiderseits lotrechten Kopfteil, einen Mittelteil, welcher an der Wasserseite noch lotrecht, an der Landseite aber nach einer einbiegenden Kurve geböscht ist, und den Fußteil, der in jener Tiefe beginnt, wo an der lotrechten Innenwand bei leerem Teiche bereits durch das Eigengewicht der Mauer der zulässige Randdruck erreicht wird und von wo ab er nicht mehr wachsen darf, sondern festwertig bleiben sollte, daher die Querschnittform am Fuße beiderseits durch allmählich sich verflachende Kurven zu begrenzen war. Im einfachsten Falle, wo die Gestalt des Unterbaues einer lotrechten Säule berechnet wird, deren Gesamthöhe größer ist als die zulässige Belastungshöhe des Baustoffes, erhält man bekanntlich logarithmische Kurven für die Begrenzung des Fußquerschnittes.

Die durch Delocre angegebene Abteilung des Mauerquerschnittes ist aber, wie aus dem Gesagten hervorgeht, unvollständig. Außerdem wollte man durch- 
weg stetige Kurven für die Stützlinien erhalten, und so gelang es nie, integrierbare Gleichungen für die Berechnung der Staumauerquerschnitte aufzustellen.

Wenn der Kopf zu einem Viadukt u. dgl. ausgebildet ist (Abb. 13), hat man dessen Gewicht und Schwerlinie zu bestimmen und die Druckmittelpunkte $m_{a}, m_{i}$ zu finden, wobei zu beachten, daß dieselben in der Linie $C D$, wo der Hals beginnt, nicht über die Kernräder hinausfallen dürfen, aber im allgemeinen eine beliebige Lage innerhalb des Kernes haben können.

Den Rumpf nehmen wir vorläufig an der Wasserseite als lotrecht an. Er sollte aber theoretisch in zwei Hälften getrennt werden. Die obere Hälfte reicht bis zur Grenzhöhe $h_{u}$ [Abb. 11 und Gl. (12)], die untere Hälfte reicht bis zur Grenzhöhe $h_{0}$, müßte aber an der Außenseite schon einer Form von gleicher Festigkeit entsprechen.

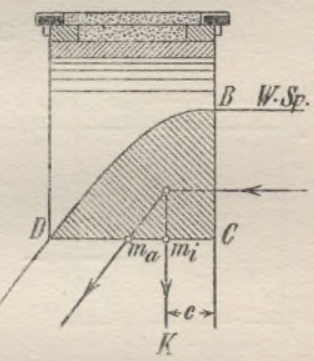

Abb. 13.

Wir erhielten also theoretisch fünf Abteilungen des Querschnittes, für deren jede andere statische Bedingungen gelten.

Es wird sich zeigen, wie weit diesen theoretischen Anforderungen praktisch entsprochen werden kann. Man könnte nach obigem die freistehenden Staumauern in zwei Gruppen einteilen:

\section{Mauern von mäßiger Höhe, Mauern von großer Höhe.}

Als Mauern von mäßiger Höhe wären solche $z u$ bezeichnen, bei denen die Grenzhöhe nicht überschritten wird, welche also aus Kopf, Hals und Rumpf bestehen. Unter Mauern von großer Höhe hätten wir jene zu 
verstehen, die höher sind als die Grenzhöhe oder zulässige Belastungshöhe und welche einen Fuß erforderten.

Die Berechnung beginnt naturgemäß mit dem Grunddreieck und dem Kopf, und es sollen nun die Bestimmungsgleichungen für die einzelnen Felder des Querschnittes der Reihe nach entwickelt werden.

\section{B. Berechnung der Abmessungen.}

a) Kopf (Abb. 14).

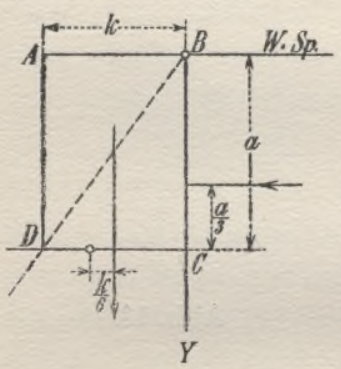

Abb. 14.

Die Gleichgewichtbedingung ist

$$
\gamma \cdot a k \cdot \frac{k}{6}=\frac{a^{3}}{6},
$$

daher

$$
\frac{k}{a}=\frac{1}{\sqrt{\gamma}} \text {. }
$$

wie beim Grunddreieck [vgl. Gl.(1)]. Die Diagonale $B D$ fällt mit der Hypotenuse des Grunddreieckes zusammen.

b) Hals.

Allgemeiner Fall. (Abb. 15).

Die Rückwand von Kopf und Hals soll in einer lotrechten Ebene liegen. Der Kopf sei beliebig ausgestaltet. Sein Gewicht, auf die Längeneinheit zurückgeführt, sei $K$, der Abstand seiner Schwerlinie von der $Y$-Achse sei $c$, seine Grundlinie $D C=k$, die Höhe von der Grundlinie $C D$ bis zum Wasserspiegel sei $a$.

Durch die Konstruktion des Kopfes sind sonach gegeben $k, a, c, K$. 
Der Hals $C D E F$ soll trapezförmig werden und seine Höhe $z$ und seine Grundlinie $f=x+k$ sind so zu bemessen, daß die Druckmittelpunkte $M_{a}$ und $M_{i}$ an die Kernränder fallen.

Die äußere Begrenzung $D E$ könnte so berechnet werden (vgl. Kressnik a. a. O.), daß zwischen den beiden Grundlinien $C D$ und $E F$ die äußere Stützlinie der Kerngrenze folgt. Macht man dagegen $D E$ geradlinig, so trifft die äußere Stützlinie nur in den beiden Grundlinien die Kerngrenze. Dazwischen tritt sie in die Kernfläche hinein. Das ist aber so geringfügig und der erzielbare Gewinn an Querschnittfläche so unbedeutend, daß wir ohne Bedenken auf diese Verfeinerung verzichten wollen.

Man berechne die dem mittleren Kopf-Querschnitt gleichwertige Fläche

$$
A_{00}=\frac{K}{\gamma} \text {. }
$$

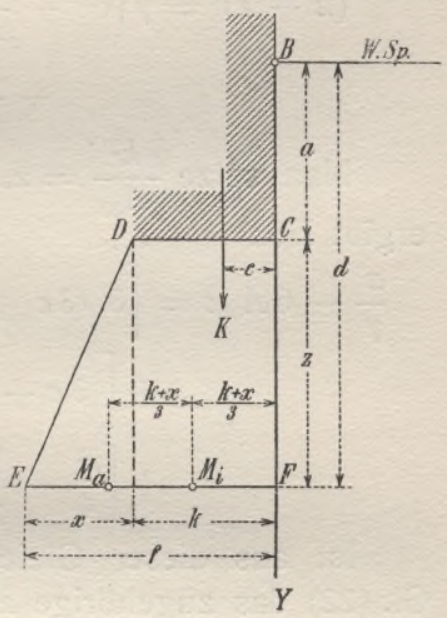

Abb. 15.

Erste Bedingung: Die Schwerlinie von Kopf und Hals zusammen soll durch $M_{i}$ gehen. Dann ist die Gleichung der statischen Momente um den Punkt $E$

$$
\begin{gathered}
\frac{2}{3}(k+x)\left[A_{00}+\frac{z}{2}(x+2 k)\right]=A_{00}(x+k-c)+ \\
+\frac{2}{3} x \cdot \frac{x z}{2}+\left(x+\frac{k}{2}\right) k z .
\end{gathered}
$$

Hieraus erhält man

$$
x=3 c-k+\frac{1}{2} \cdot \frac{z k^{2}}{A_{00}} .
$$


Zweite Bedingung: Die Mittelkraft aus dem Gewichte von Kopf und Hals und dem Wasserdruck auf die Ebene $B F$ soll durch den äußeren Drittelspunkt $M_{a}$ der Grundlinie $E F$ gehen.

$$
\frac{(a+z)^{3}}{6}=\gamma \frac{k+x}{3}\left[A_{00}+\frac{z}{2}(x+2 k)\right] .
$$

Führt man $x$ aus Gl. (22) ein, so erhält man

$$
\begin{aligned}
(a+z)^{3}=\gamma(6 c & \left.+\frac{z k^{2}}{A_{00}}\right)\left[A_{00}+\frac{z}{2}(3 c+k)+\right. \\
& \left.+\frac{z^{2}}{4} \cdot \frac{k^{2}}{A_{00}}\right] . . . . .
\end{aligned}
$$
ergibt sich

Man setze $\frac{k^{2}}{A_{00}}=x$, entwickle und ordne, dann

$$
\begin{aligned}
\frac{a^{3}}{\gamma}-6 A_{00} c= & {\left[3(3 c+k) c+k^{2}-\frac{3 a^{2}}{\gamma}\right] z+} \\
& +\left[\left(3 c+\frac{k}{2}\right) x-\frac{3 a}{\gamma}\right] z^{2}+ \\
& +\left[\frac{x^{2}}{4}-\frac{1}{\gamma}\right] z^{3} . . . . . .
\end{aligned}
$$

Ist aus dieser Gleichung $z$ bestimmt, so ergibt Gl. (22) das zugehörige $x$.

$$
\text { Besonderer (einfachster) Fall: }
$$

Kopf rechteckig, bis an den Wasserspiegel reichend.

Hier ist $A_{00}=a k=k^{2} \sqrt{\gamma}(\mathrm{Gl} .21), c=\frac{k}{2}$, daher nach Gl. (22)

$$
x=\frac{3}{2} k-k+\frac{1}{2} \cdot \frac{z k^{2}}{k^{2} \sqrt{\gamma}}=\frac{k}{2}+\frac{z}{2 \sqrt{\gamma}}
$$

oder, da $k=\frac{a}{\sqrt{\gamma}}$

$$
x=\frac{a+z}{2 \sqrt{\gamma}} \ldots . . .
$$


und Gl. (23) geht über in

$$
(a+z)^{3}=\gamma\left(\frac{3 a+z}{\sqrt{\gamma}}\right)\left[\frac{a^{2}}{\sqrt{\gamma}}+\frac{z}{2} \cdot \frac{5 a}{2 \sqrt{\gamma}}+\frac{z^{2}}{4 \sqrt{\gamma}}\right]
$$

woraus sich entwickelt

$$
(a+z)^{2}=(3 a+z)\left(a+\frac{1}{4} z\right)
$$

oder

$$
8 a^{2}=a z+3 z^{2},
$$

wonach

$$
z=\frac{a}{6}(-1+\sqrt{97}),
$$

daher

$$
\begin{aligned}
& \frac{z}{a}=1,475 \\
& \frac{z}{k}=1,475 \sqrt{\gamma} \\
& \frac{x}{k}=\frac{a+z}{2 a}=1,237
\end{aligned}
$$

ferner

$$
\begin{aligned}
& a+z=d=2,475 a=2,475 k \sqrt{\gamma} \\
& k+x=f=2,237 k
\end{aligned}
$$

und die Querschnittfläche von Kopf und Hals zusammen

$$
A_{0}=3,387 \sqrt{\gamma} \cdot k^{2}
$$

c) Rumpf, obere Hälfte (Abb. 16).

Bis zur Höhe $y=h_{n}$ [Gl. (12)] gilt die Bedingung, $\mathrm{da} \beta$ an der Wasserseite bei gefülltem Teich überall $\beta_{m}=0$ sein muß, weshalb aus Gl. (11) sich ergibt

$$
G t=62 \Re
$$

und, wenn man $G$ aus (7), $\mathfrak{i}$ aus (8) einführt

$$
\gamma t \int_{0}^{y} t d y=y^{3}+3 \gamma\left(\int_{0}^{y} t^{2} d y-t \int_{0}^{y} t d y\right)
$$

oder

$$
4 \gamma t \int_{0}^{y} t d y=y^{3}+3 \gamma \int_{0}^{y} t^{2} d y
$$


Es ist aber

$\int_{0}^{y} t d y$ die Fläche des Querschnittes von der Krone bis zur Wagerechten in der Tiefe $y$, $\int_{0}^{y} t^{2} d y$ das doppelte statische Moment dieser Fläche um die $Y$-Achse.

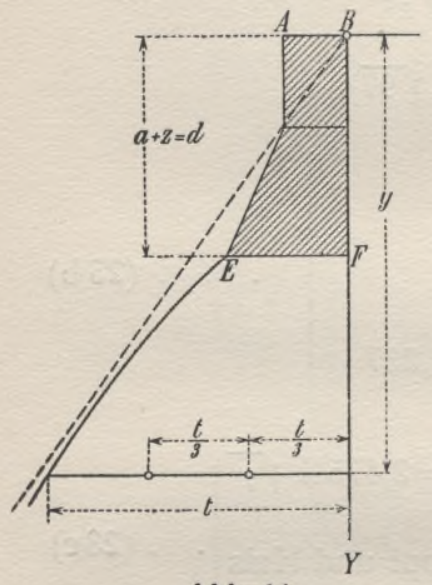

Abb. 16.

so ist

Bezeichnen wir daher

$$
\begin{aligned}
& \int_{0}^{y} t d y \text { durch } A, \\
& \int_{0}^{y} t^{2} d y \text { durch } 2 x,
\end{aligned}
$$

$$
\mathfrak{u}=\frac{2}{3} t \cdot A,
$$

daher nach Gl. (24)

$$
\begin{gathered}
4 \gamma t A=y^{3}+2 \gamma t A \\
t=\frac{y^{3}}{2 \gamma A} . .
\end{gathered}
$$

weil aber $t d y=d A, t=\frac{d A}{d y}$, so hat man

$$
2 A d A=\frac{1}{\gamma} y^{3} d y
$$

mithin

$$
A^{2}=\frac{y^{4}}{4 \gamma}+C
$$

Die Fläche von Kopf und Hals zusammen haben wir bereits berechnet und durch $A_{0}$ bezeichnet.

Für $y=d$ wird $A=A_{0}$, daher $C=A_{0}^{2}-\frac{d^{4}}{4 \gamma}$ und

$$
A^{2}=\frac{y^{4}-d^{4}}{4 \gamma}+A_{0}^{2} \ldots . .
$$

und, wenn man den hierdurch erhaltenen Wert von $A$ in die Gleichung (25) einführt, erhält man

$$
t=\frac{y^{3}}{\sqrt{\gamma\left(y^{4}-d^{4}+4 \gamma A_{0}^{2}\right)}} . . .
$$


Diese Gleichung liefert also, für Werte von $y$ zwischen den Grenzen $d$ und $h_{u}$, die Breiten $t$.

Die Rechnung wird einfacher, wenn man in den Ausdruck (25a) die Werte von $d$ und $A_{0}$ aus (23c) einführt, wonach sich für die Ausübung ergibt

$$
\begin{aligned}
& t=\frac{y^{3}}{\sqrt{\gamma\left(y^{4}+8,365 \gamma^{2} k^{4}\right)}} \ldots . \\
& A=\frac{1}{2 \sqrt{\gamma}} \sqrt{y^{4}+8,365 \gamma^{2} k^{4}}
\end{aligned}
$$

Für $k=0$ erhält man aus (25a) und (25b) den für das Grunddreieck gültigen Ausdruck (1).

Für $k>0$ sind also die Breiten $t$ stets kleiner als beim Grunddreieck und die Kurve der äußeren Böschung nähert sich asymptotisch der Hypotenuse des Grunddreieckes.

Je schwerer das Gemäuer, desto schmäler wird das Profil - was sich eigentlich von selbst versteht. Schweres Gemäuer ist - gleichen Preis vorausgesetzt - wirtschaftlich vorteilhaft.

Hiermit ist aber zunächst nur die Bedingung erfüllt, daß die beiden Stützlinien im Rumpfe durchweg im Abstande $\frac{t}{3}$ voneinander verlaufen, und zwar unter der Voraussetzung, daß die dem Wasserdrucke ausgesetzte Fläche als lotrecht, der Wasserdruck durchweg als wagerecht angenommen werden dürfe.

Wenn man einen Staumauerquerschnitt nur nach den im vorstehenden entwickelten Formeln berechnet und mit durchweg lotrechter innerer Wand aufträgt, so wird die äußere Stützlinie im Rumpfe ein wenig in die Kernfläche herein, die innere um ebensoviel aus ihr hinausrücken, so daß also bei vollem Teiche die Beanspruchung etwas günstiger wird, bei leerem Teiche aber 
an der äußeren Böschung kleine Zugspannungen auftreten. Diese sind aber so geringfügig, daß man sie bei niedrigen Talsperren (z. B. bis zu $15 \mathrm{~m}$ Höhe) kaum zu berücksichtigen braucht, und auch in Fällen, wo der Wasserspiegel nicht regelmäßig und beträchtlich schwankt, wie bei Wildbachsperren, die innere Wand unbedenklich lotrecht machen könnte.

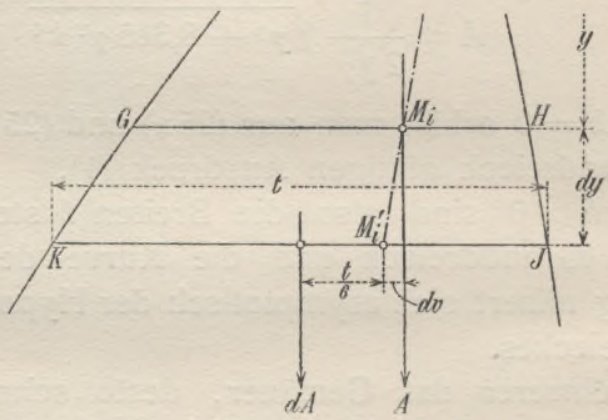

Abb. 17.

Für große Talsperren aber, die zur Bildung künstlicher Seen zu dienen haben, darf man die Mühe einer vollständigen Berechnung nicht scheuen.

Noch zu erfüllen ist also die Bedingung, daß für jede wagerechte Schnittlinie der Schwerpunkt des über ihr liegenden Teiles der Querschnittfläche lotrecht über dem hinteren Drittelspunkte seiner Grundlinie liege.

Diese Bedingung stellt sich folgendermaßen dar (Abb. 17):

$A$ sei die gesamte Querschnittfläche über der Wagerechten $H G$, die in der Tiefe $y$ unter der Krone liegt. Die Schwerlinie von $A$ gehe durch den inneren Dreiteilungspunkt $M_{i}$ von $G H$.

Läßt man nun $y$ um $d y$ zunehmen, so wächst $A$ um $d A$ und die neue Schwerlinie muß durch den inneren 
Dreiteilungspunkt $M^{\prime}{ }_{i}$ der neuen Grundlinie $J K$ gehen, welcher gegen den vorigen um $d v$ seitlich verschoben ist. Es muß also die Gleichgewichtbedingung erfüllt sein:

$$
d A \cdot \frac{t}{6}=A d v
$$

oder, da $d A=t d y$

$$
\frac{t^{2}}{6} d y=A d v \text {. }
$$

Wir führen für $t$ den Ausdruck (25) ein und erhalten

$$
d v=\frac{t^{2}}{6 A} d y=\frac{y^{6}}{24 \gamma^{2} A^{3}} d y .
$$

Nach Gl. (26) ist aber

$$
\begin{aligned}
& A^{3}=\left(\frac{y^{4}-d^{4}}{4 \gamma}+A_{0}^{2}\right)^{3 / 8}= \\
& =\frac{1}{8 \gamma^{3 / 8}}\left(y^{4}-d^{4}+4 \gamma A_{0}^{2}\right)^{3 / 2} .
\end{aligned}
$$

Festwert

Setzt man [vgl. $(25 \mathrm{~b})]$ den

$$
-d^{4}+4 \gamma A_{0}{ }^{2}=8,365 \gamma^{2} k^{4}=\Phi,
$$

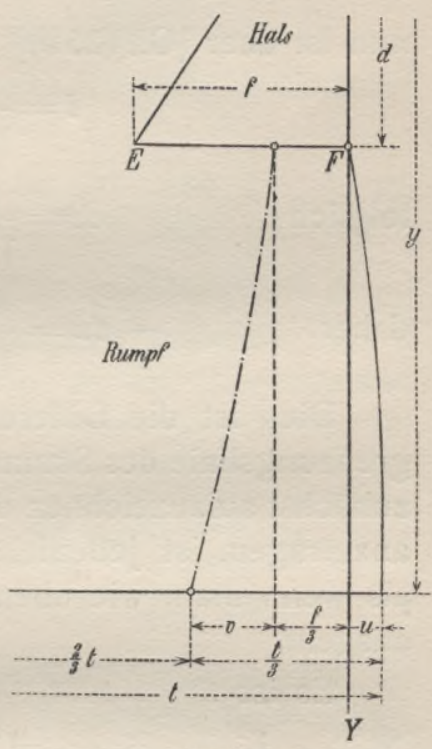

Abb. 18.

so folgt

$$
d v=\frac{1}{3 \sqrt{\gamma}} \cdot \frac{y^{6}}{\left(y^{4}+\Phi\right)^{3 / 2}} d y \quad . \quad .
$$

Dies ist die Differentialgleichung der inneren Stützlinie für den Rumpf.

Der Ursprung dieser Kurve ist der innere Dreiteilungspunkt der Grundlinie $E F$ des Halses (Abb. 18).

Hätte man für irgendeine Tiefe $y$ die wagerechte Ordinate $v$ dieser Stützlinie berechnet und aufgetragen, also den inneren Druckmittelpunkt für die betreffende Wagerechte gefunden, so wäre von da aus ${ }^{1} / 3 t$ nach innen und ${ }^{2} t$ nach außen aufzutragen und die innere Be- 
grenzung des Staumauerquerschnittes würde um ein Stück $u$ über die Grundachse $Y$ hinausrücken, so daß

$$
-u=\frac{t}{3}-\frac{f}{3}-v \quad \text { oder } \quad-d u=\frac{1}{3} d t-d v,
$$

nun ist aber [Gl. (25b)]

$$
t=\frac{y^{3}}{\sqrt{\gamma}\left(y^{4}+\Phi\right)^{1 / 2}}
$$

folglich

$$
d t=\frac{1}{\sqrt{\gamma}} \cdot \frac{y^{6}+3 y^{2} \Phi}{\left(y^{4}+\Phi\right)^{3 / 2}} d y
$$

daher

$$
-d u=\frac{\Phi}{\sqrt{\gamma}} \cdot \frac{y^{2}}{\left(y^{4}+\Phi\right)^{3 / 2}} \cdot d y .
$$

Dies ist die Differentialgleichung der inneren Begrenzungslinie des Staumauerquerschnittes. Diese Kurve zunächst aufzuzeichnen und von ihr herüber die Breiten $t$ anzutragen, ist jedenfalls für die Ausübung bequemer, als wenn man, wie oben angedeutet, von der Stützlinie ausgeht.

Es ist

$$
\frac{\Phi}{\sqrt{\gamma}} \cdot \frac{y^{2}}{\left(y^{4}+\Phi\right)^{3 / 2}}=\frac{y^{2}}{y^{6} \cdot\left(1+\frac{\Psi}{y^{4}}\right)^{3 / 2}} \cdot \frac{\Phi}{\sqrt{\gamma}}=\left[\frac{\Phi}{y^{4}} \cdot \frac{1}{\left(1+\frac{\Phi}{y^{4}}\right)^{3 / 2}}\right] \frac{1}{\sqrt{\gamma}}
$$

Der eingeklammerte Ausdruck hat die Form

$$
\frac{x}{(1+x)^{n}}=x(1+x)^{-n} \text {. }
$$

Für $n=\frac{3}{2}$ erhält man nach dem binomischen Lehrsatz:

$$
\begin{aligned}
(1+x)^{-3 / 2} & =1-\frac{3}{2} x+\frac{3 \cdot 5}{2^{2} \cdot 1 \cdot 2} x^{2}-\frac{3 \cdot 5 \cdot 7}{2^{3} \cdot 1 \cdot 2 \cdot 3} x^{3}+\frac{3 \cdot 5 \cdot 7 \cdot 9}{2^{4} \cdot 1 \cdot 2 \cdot 3 \cdot 4} x^{4}-. \\
& =1-\frac{3}{2} x+\frac{15}{8} x^{2}-\frac{105}{48} x^{3}+\frac{945}{384} x^{4}-\ldots
\end{aligned}
$$


daher

$$
\begin{aligned}
-d u & =\frac{1}{\sqrt{\gamma}} \cdot \frac{\Phi}{y^{4}}\left[1-\frac{3}{2}\left(\frac{\Phi}{y^{4}}\right)+\frac{15}{8}\left(\frac{\Phi}{y^{4}}\right)^{2}-\frac{105}{48}\left(\frac{\Phi}{y^{4}}\right)^{3}+\frac{945}{384}\left(\frac{\Phi}{y^{4}}\right)^{4}-\cdots\right] \\
& =\frac{\Phi}{\sqrt{\gamma}}\left[\frac{1}{y^{4}}-\frac{3}{2} \frac{\Phi}{y^{8}}+\frac{15}{8} \frac{\Phi^{2}}{y^{12}}-\frac{105}{48} \frac{\Phi^{3}}{y^{16}}+\frac{945}{384} \frac{\Phi^{4}}{y^{20}}-\ldots\right],
\end{aligned}
$$

und durch Integration und teilweise Kürzung der Brüche erhält man

$$
u=-\frac{\Phi}{\sqrt{\gamma}}\left(-\frac{1}{3 y^{3}}+\frac{3}{14} \cdot \frac{\Phi}{y^{7}}-\frac{15}{88} \cdot \frac{\Phi^{2}}{y^{11}}+\frac{35}{240} \cdot \frac{\Phi^{3}}{y^{15}}-\frac{315}{2432} \cdot \frac{\Phi^{4}}{y^{19}}+\cdots+C\right)
$$

Da $u=0$ für $y=d$, so ist der Festwert $C$ zu erhalten, indem man die Vorzeichen umkehrt und $d$ statt $y$ einführt. Die Rechnung ist mit Logarithmen leicht durchführbar, und wir schreiben daher in der für diesen $Z$ weck bequemeren Form

$C \cdot \Phi=\frac{\frac{1}{3} \Phi}{d^{3}}-\frac{\frac{3}{14} \Phi^{2}}{d^{7}}+\frac{\frac{15}{88} \Phi^{3}}{d^{11}}-\frac{\frac{35}{240} \Phi^{4}}{d^{15}}+\frac{\frac{315}{2432} \Phi^{5}}{d^{19}}-\cdots$

Wie man sich leicht überzeugt, konvergiert die Reihe rasch. Will man $u$ auf Zentimeter ausrechnen, so geht man nur bis zu jenem Gliede, welches Millimeter ergibt. Meist werden die ersten fünf Glieder genügen für die Konstante. Für den veränderlichen Teil der $u$, also für $y>d$, braucht man immer weniger Glieder, je größer $y$ wird. Zuletzt genügt bei hohen Mauern das erste Glied.

Man erhält somit

$$
\begin{array}{r}
u=-\frac{1}{\sqrt{\gamma}}\left(-\frac{\frac{1}{3} \Phi}{y^{8}}+\frac{\frac{3}{14} \Phi^{2}}{y^{7}}-\frac{\frac{15}{88} \Phi^{3}}{y^{11}}+\frac{\frac{35}{240} \Phi^{4}}{y^{15}}-\cdots\right. \\
\cdots+C \cdot \Phi) . . . . .
\end{array}
$$


$\mathrm{Da}$ der Ausdruck für $t$ [Gl.(25)] gefunden wurde unter der Voraussetzung, daß der Wasserdruck überall wagerecht, also die innere Wand lotrecht sei, so wird bei einer, wie vorstehend berechneten Staumauer die äußere Stützlinie, welche dem gefüllten Teiche entspricht, nicht genau an der äußeren Kerngrenze verlaufen, sondern ganz wenig in die Kernfläche hereinrücken. Das ist aber günstig.

Führt man dagegen eine Schirmmauer aus, die innen lotrecht ist, was sich ganz naturgemäß ergibt, so überträgt diese mittels der Rippen den Wasserdruck wagerecht auf die Staumauer und die Ergebnisse der Berechnung entsprechen genau den Voraussetzungen.

Wenn man der Schirmmauer außen Absätze gibt, um ihre Dicke nach der Tiefe zunehmen zu lassen, so ändert dies an der Sache nichts.

d) Rumpf, untere Hälfte (vgl. Abb. 11, S. 27).

Nach Gl. (10) ist

$$
t^{2} \beta_{n}=G t+62 \pi \text {. }
$$

Die zweite Bedingung lautet

$$
O=G t-62 \pi \text {, }
$$

woraus

$$
\begin{aligned}
& t^{2} \beta_{n}=2 G t \\
& t^{2} \beta_{n}=2 \gamma t \int_{0}^{y} t d y .
\end{aligned}
$$

$\beta_{n}$ müßte eigentlich veränderlich sein, wenn die Böschung sich ändert. Da die Berücksichtigung dieser Forderung große Schwierigkeiten macht, so wollen wir als Näherungswert denjenigen einführen, welcher der Höhe $h_{u}$ entspricht, wonach $h_{u}=\frac{\beta_{n}}{\gamma}$ und wir somit $\beta_{n}$ 
für ein $y$, das nicht viel größer ist, als $h_{u}$, festwertig annehmen. Dann ist

differenziert

$$
t^{2} h_{n}=2 t \int_{0}^{y} t d y, t h_{u}=2 \int_{0}^{y} t d y,
$$

$$
d t=\frac{2}{h_{u}} \cdot t d y, \text { folglich } \ln t=\frac{2}{h_{u}} y+C .
$$

Für $y=h_{u}$ erhält man nach Gl. (25a) eine Breite $t_{u}$, daher

wonach

$$
\ln t_{u}=\frac{2}{h_{u}} h_{u}+C,
$$

$$
\begin{aligned}
\ln \frac{t}{t_{u}} & =\frac{2}{h_{u}} y-2=\frac{2}{h_{u}}\left(y-h_{u}\right) \\
t & =t_{u} e^{\frac{2}{h_{u}}\left(y-h_{u}\right)}
\end{aligned}
$$

Aus praktischen Rücksichten setzen wir fest, daß die Böschung nicht flacher werden darf als $1: 1$, da sonst der Fuß zu schwach wird an der Außenkante (oder, wie der Engländer treffend sagt, an der "Zehe“ - toe) und überdies die Zulässigkeit der Trapezregel sehr fraglich wird.

Diese Bedingung wäre

$$
\frac{d t}{d y} \leqq 1 .
$$

Obiger Ausdruck für $t$ ergibt

$$
\frac{d t}{d y}=t_{u} \frac{2}{h_{u}} e^{\frac{2}{h_{u}}\left(y-h_{u}\right)}
$$

und es müßte sein

$$
\begin{gathered}
\ln \frac{2 t_{u}}{h_{u}}+\frac{2}{h_{u}}\left(y-h_{u}\right) \ln e=0 \\
\frac{2}{h_{u}}\left(y-h_{u}\right)=-\frac{\ln \frac{2 t_{u}}{h_{u}}}{\ln e}
\end{gathered}
$$




$$
\begin{gathered}
y=h_{u}-\frac{h_{u}}{2} \cdot \frac{\ln \frac{2 t_{u}}{h_{u}}}{\ln e} \\
y<h_{u},
\end{gathered}
$$

d. h. für $y>h_{n}$ wird $\frac{d t}{d y}$ jedenfalls $>1$, daher die angenommene praktische Grenze überschritten. Der untere Teil des Rumpfes ist somit praktisch unzulässig, es verbleibt bei der Einteilung der Querschnittfläche in nur vier Felder und der Rumpf hat seine Grenze in der Tiefe $h_{u}$.

e) Fufs (Abb. 19).

1. Entwickelung der Grundgleichungen.

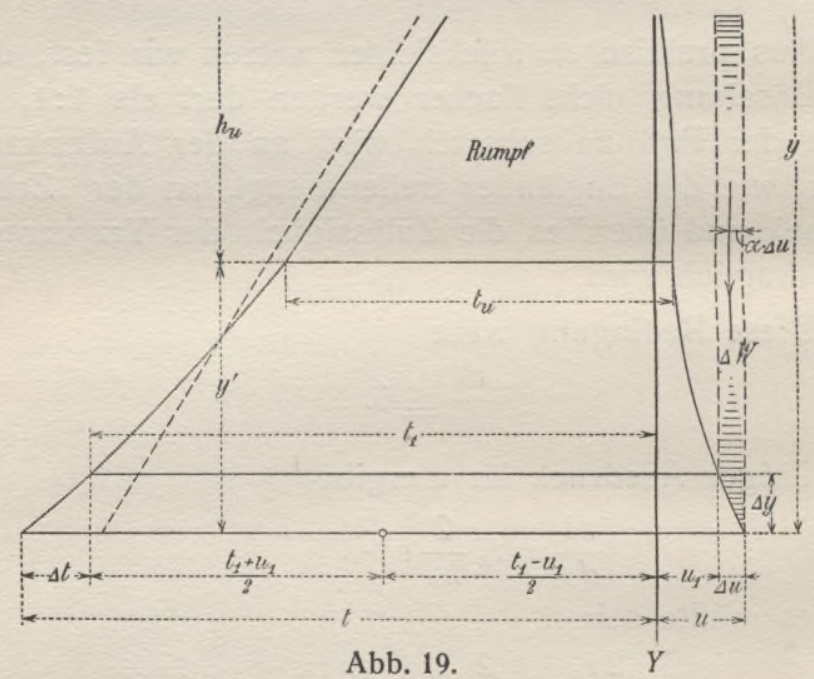

Im Rumpfe hat die Randspannung mit der Tiefe stetig zugenommen.

Im Fuße soll sie festwertig werden. 
Es muß daher an der Grenze zwischen Rumpf und Fuß eine Unstetigkeit auftreten.

Man erkennt sofort, daß man keinen Fuß mehr anwenden kann, wenn man mit der Höhe des Rumpfes bereits bis an die Grenze gegangen ist. Denn, wenn $\beta_{n}$ unveränderlich bleiben soll, so müssen die Böschungen sich verflachen, somit wird $\varepsilon$ wachsen und, nach (Gl. $3 \mathrm{a})$, die Scherspannung rasch zunehmen.

Man hätte also von Fall zu Fall zu untersuchen, ob sich eine nennenswert günstigere Beanspruchung auf Abscheren an der äußeren Böschung - die innere kommt kaum in Betracht - erzielen läßt, wenn man mit dem Fuße etwas weiter oben beginnt. Da man aber sich nicht $\mathrm{zu}$ weit vom Grunddreieck entfernen darf, wenn nicht die Hauptgrundlage der Rechnung die Trapezregel - hinfällig werden soll, so folgt, daß die Anwendung eines Fußes jedenfalls sehr beschränkt ist und bei den meisten Ausführungen in bedenklichem Maße übertrieben wurde.

Nichtsdestoweniger soll der Vollständigkeit halber eine unmittelbare Berechnungsweise für den Fuß hier vorgeführt werden.

Bei gefülltem Teiche hat an der Außenseite, bei leerem an der Innenseite, die Stärke des lotrecht gemessenen Randruckes unveränderlich gleich $\beta_{n} \mathrm{zu}$ bleiben.

Die Grundgleichungen sind:

1. Für den Fall, daß der Teich bis zur Kronenkante gefüllt ist

$$
\text { Gl. (16a) }(t+u)^{2} \max \beta_{n}=(t+u) P+62 \Re
$$

2. Für den Fall, daß der Teich leer ist, $\max \beta_{n}$ statt $\max \beta_{m}$ gesetzt,

Gl. (19a) $(t+u)^{2} \max \beta_{n}=(t+u) G+62 \tau$. 
Daher erhalten wir

1. bei gefülltem Teiche mittels (13) und (14):

$$
\begin{aligned}
(t+u)^{2} \beta_{n}= & (t+u)\left[\gamma \int_{0}^{y}(t+u) d y+\int_{0}^{y} y d u\right]+y^{3}+ \\
& +3 \gamma\left[\int_{0}^{y}\left(t^{2}-u^{2}\right) d y-(t-u) \int_{0}^{y}(t+u) d y\right]- \\
& -6 \int_{0}^{y} u y d u-3(t-u) \int_{0}^{y} y d u
\end{aligned}
$$

oder, zusammengezogen,

$$
\begin{aligned}
(t+u)^{2} \beta_{n}= & y^{3}-2(t-2 u)\left[\gamma \int_{0}^{y}(t+u) d y+\int_{0}^{y} y d u\right]+ \\
& +3\left[\gamma \int_{0}^{y}\left(t^{2}-u^{2}\right) d y-2 \int_{0}^{y} u y d u\right] . .
\end{aligned}
$$

Wir bezeichnen nun die Fläche des Querschnittes, welche über der Grenzlinie liegt, also der Höhe $h_{u}$ entspricht, durch $A_{u}$. Wir erhalten sie aus Gl. (26), indem wir $h_{u}$ für $y$ einführen.

Das doppelte statische Moment der Fläche $A_{u}$ um die $Y$-Achse bezeichnen wir $\mathfrak{U}_{u}$.

Die Fläche für den $\mathrm{FuB}$, bis zur Gesamttiefe $y=h_{u}+y^{\prime}$, oder von der Grenze bis auf die Tiefe $y^{\prime}$ unter ihr, sei $B$; ihr doppeltes statisches Moment um die $Y$-Achse sei $\mathfrak{B}$.

Die Fläche $\int_{0}^{y} y d u$ des Wasserkörpers, welcher den Fuß belastet, sei $W$, und $\int_{0}^{y} u y d u$, ihr e infaches statisches Moment um die $Y$-Achse, sei $\mathfrak{W}$.

Dann erhält Gl. (30) die übersichtlichere Form:

$$
\begin{array}{r}
(t+u)^{2} \beta_{n}=\left(\mathrm{h}_{u}+y^{\prime}\right)^{3}-2(t-2 u)\left[\gamma\left(A_{u}+B\right)+W\right]+ \\
+3\left[\gamma\left(\mathfrak{U}_{u}+\mathcal{B}\right)-2 \mathfrak{W}\right] . . \quad(30 \mathrm{a})
\end{array}
$$


2. Bei leerem Teiche müssen wir haben, nach Gl. (19a), (17) und (18)

$$
\begin{aligned}
& (t+u)^{2} \beta_{n}=(t+u) \gamma \int_{0}^{y}(t+u) d y+ \\
& +3 \gamma\left[(t-u) \int_{0}^{y}(t+u) d y-\int_{0}^{y}\left(t^{2}-u^{2}\right) d y\right]
\end{aligned}
$$

oder, zusammengezogen,

$$
(t+u)^{2} \beta_{n}=2(2 t-u) \gamma \int_{0}^{y}(t+u) d y-3 \gamma \int_{0}^{y}\left(t^{2}-u^{2}\right) d y(31)
$$

und wenn man für die Flächen und deren Momente die vorigen Bezeichnungen einführt

$$
(t+u)^{2} \beta_{n}=2(2 t-u) \gamma\left(A_{u}+B\right)-3 \gamma\left(\mathfrak{Z}_{u}+\mathcal{B}\right)
$$

Man berechnet nun absatzweise das $t$ und $u$, wie folgt:

Für eine Schichte von mäßiger Höhe $\Delta y$ kann man die beiden Böschungen als geradlinig annehmen. Dann hat man für diese Schichte, mit den aus Abb. 19 ersichtlichen Bezeichnungen, unter der Voraussetzung, daß für deren obere Begrenzung die Breiten $t_{1}, u_{1}$ bereits gefunden, $B_{1}, \mathfrak{B}_{1}, W_{1}, \mathfrak{W}_{1}$, berechnet seien

$$
\begin{aligned}
& W=W_{1}+\Delta W, \\
& \mathfrak{W}=\mathfrak{W}_{1}+\Delta W(u-\alpha \Delta u) .
\end{aligned}
$$

$\alpha \Delta u$ (Abb. 20) ist der Abstand der Schwerlinie des Trapezes $\Delta W$ von der inneren (längeren) Grundlinie, daher,

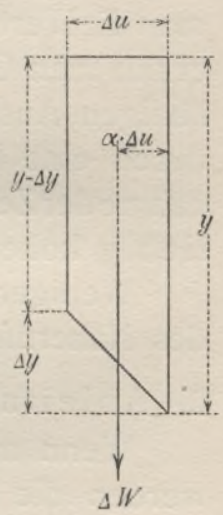

Abb. 20. nach bekannter Formel

$$
\begin{aligned}
\alpha \cdot \Delta u & =\frac{\Delta u}{3} \cdot \frac{y+2(y-\Delta y)}{y+(v-\Delta y)} \\
\alpha & =\frac{1}{3} \cdot \frac{3 y-2 \Delta y}{2 y-\Delta y} .
\end{aligned}
$$

Kreuter, Staumauern. 
Ferner ist $B=B_{1}+\Delta B, \mathfrak{B}=\mathfrak{B}_{1}+\Delta \mathfrak{B}$, und zwar findet man nach der Abb. 19

$$
\begin{aligned}
\Delta B= & \left(t_{1}+u_{1}\right) \Delta y+\frac{1}{2}(\Delta t+\Delta u) \Delta y \\
\Delta \mathcal{B}= & \left(t_{1}{ }^{2}-u_{1}^{2}\right) \Delta y+\Delta t \cdot \Delta y\left(t_{1}+\frac{\Delta t}{3}\right)- \\
& -\Delta u \cdot \Delta y\left(u+\frac{\Delta u}{3}\right) \\
\Delta W= & y \Delta u-\frac{1}{2} \Delta u \cdot \Delta y \\
\Delta \mathfrak{W}= & {\left[u_{1}+(1-\alpha) \Delta u\right]\left(y \Delta u-\frac{1}{2} \Delta u \cdot \Delta y\right) }
\end{aligned}
$$

Außerdem haben wir

$$
t=t_{1}+\Delta t_{1}, \quad u=u_{1}+\Delta u . . .
$$

Die Unbekannten sind $\Delta t$ und $\Delta u$, die wir aus den Gleichungen ( $30 \mathrm{a})$ und (31a) zu bestimmen haben. Führt man die Werte aus (33) und (33a) in (30a) und (31a) ein, so erhält man Gleichungen höherer Ordnung mit sehr weitläufigen Ausdrücken für die Koeffizienten, wonach sich, durch eine Folge von Versuchrechnungen mit Zuhilfenahme der Zeichnung, die Ausladungen $\Delta t$ und $\Delta u$ für beliebig hohe, trapezförmige Schichten berechnen lassen.

Weniger ermüdend und übersichtlicher dürfte folgendes Näherungsverfahren sein:

2. Ableitung der Bestimmungsgleichungen.

Wenn man die Schichten dünn genug macht, dann werden die Differenzen $\Delta y, \Delta t, \Delta u$ so klein im Verhältnis zu $y, t, u$, daß man die Potenzen und Produkte der Differenzen vernachlässigen kann. Man erhält dann annähernd:

$$
\left.\begin{array}{l}
\Delta B=\left(t_{1}+u_{1}\right) \Delta y \\
\Delta \mathcal{B}=\left(t_{1}^{2}-u_{1}\right)^{2} \Delta y \\
\Delta W=y \cdot \Delta u=\left(h_{u}+y^{\prime}\right) \Delta u \\
\Delta \mathfrak{W}=u_{1} \cdot y \cdot \Delta u=\left(h_{u}+y^{\prime}\right) u_{1} \Delta u
\end{array}\right\}
$$


Dies in Gleichungen (30a) eingeführt, ergibt

$$
\left[\left(t_{1}+u_{1}\right)^{2}+2\left(t_{1}+u_{1}\right)(\Delta t+\Delta u)\right] \beta_{n}=\left(h_{n}+y^{\prime}\right)^{3}-
$$

$-2\left[\left(t_{1}-2 u_{1}\right)+(\Delta t-2 \Delta u)\right] \cdot\left\{\gamma\left[A_{u}+B_{1}+\left(t_{1}+u_{1}\right] \Delta y\right]+W_{1}+\left(h_{u}+y^{\prime}\right) \Delta u\right\}+$

$+3\left\{\gamma\left[\mathfrak{U}_{u}+\mathfrak{B}_{1}+\left(t_{1}{ }^{2}-u_{1}{ }^{2}\right) \Delta y\right]-2 \mathfrak{W}_{1}-2\left(h_{u}+y^{\prime}\right) u_{1} \Delta u\right\}$.

Man rechne gleich aus :

$$
\begin{aligned}
A_{u}+B_{1}+\left(t_{1}+u_{1}\right) \Delta y & =F=A_{u}+B \\
\mathfrak{U}_{u}+\mathfrak{B}_{1}+\left(t_{1}^{2}-u_{1}^{2}\right) \Delta y & =\mathcal{f}=\mathfrak{U}_{u}+\mathfrak{B},
\end{aligned}
$$

teile die ganze Gleichung durch $\gamma$ und setze $\frac{\beta_{n}}{\gamma}=h_{u}$;

$$
\begin{aligned}
& {\left[\left(t_{1}+u_{1}\right)^{2}+2\left(t_{1}+u_{1}\right)\left(\Delta t+\Delta_{u}\right)\right] h_{u}=\frac{\left(h_{u}+y^{\prime}\right)^{3}}{\gamma}-} \\
- & 2\left[\left(t_{1}-2 u_{1}\right)+(\Delta t-2 \Delta u)\right] \cdot\left[F+\frac{W_{1}}{\gamma}+\frac{h_{u}+y^{\prime}}{\gamma} \Delta u\right]+ \\
+ & 3\left[f-\frac{2 \mathfrak{W}_{1}}{\gamma}-\frac{2\left(h_{u}+y^{\prime}\right) u_{1}}{\gamma} \Delta u\right] . . . . .
\end{aligned}
$$

\section{geordnet:}

$$
\begin{gathered}
h_{u}\left(t_{1}+2 u_{1}\right)^{2}-\frac{\left(h_{u}+y^{\prime}\right)^{3}}{\gamma}+2\left(t_{1}-2 u_{1}\right)\left(F+\frac{W_{1}}{\gamma}\right)-3\left(f-\frac{2 \mathfrak{W}_{1}}{\gamma}\right)= \\
=-2\left[\left(t_{1}+u_{1}\right) h_{n}+\left(F+\frac{W_{1}}{\gamma}\right)\right] \Delta t+ \\
+2\left[2\left(F+\frac{W_{1}}{\gamma}\right)-\left(t_{1}+2 u_{1}\right) \frac{h_{u}+y^{\prime}}{\gamma}-\left(t_{1}+u_{1}\right) h_{u}\right] \Delta u \ldots \\
(34 \mathrm{a})
\end{gathered}
$$

Man rechne aus:

$$
\begin{gathered}
\frac{1}{2}\left[\left(t_{1}+u_{1}\right)^{2} h_{u}-\frac{\left(h_{u}+y^{\prime}\right)^{3}}{\gamma}+2\left(t_{1}-2 u_{1}\right)\left(F+\frac{W_{1}}{\gamma}\right)-3\left(\mathfrak{f}-\frac{2 \mathfrak{W}_{1}}{\gamma}\right)\right]=\mathfrak{D} \\
{\left[\left(t_{1}+u_{1}\right) h_{u}+F+\frac{W_{1}}{\gamma}\right]=E} \\
{\left[2\left(F+\frac{W_{1}}{\gamma}\right)-\left(t_{1}+2 u_{1}\right) \frac{h_{u}+y^{\prime}}{\gamma}-\left(t_{1}+u_{1}\right) h_{u}\right]=H .}
\end{gathered}
$$

Dann lautet die Gleichung (34a):

$$
\begin{gathered}
\mathfrak{D}=-E \cdot \Delta t+H \cdot \Delta u \\
\Delta u=\frac{\mathfrak{D}+E \cdot \Delta t}{H} .
\end{gathered}
$$


Ebenso entwickeln wie die Gleichung (31 a):

$$
\begin{aligned}
& {\left[\left(t_{1}+u_{1}\right)^{2}+2(\Delta t+\Delta u)\left(t_{1}+u_{1}\right)\right] h_{u}=} \\
& \quad=2\left[\left(2 t_{1}-u_{1}\right)+(2 \Delta t-\Delta u)\right] F-3 f \ldots \\
& \left(t_{1}+u_{1}\right)^{2} h_{u}-2\left(2 t_{1}-u_{1}\right) F+3 f= \\
& \quad=2\left[2 F-\left(t_{1}+u_{1}\right) h_{u}\right] \Delta t-2\left[F+\left(t_{1}+u_{1}\right) h_{n}\right] \Delta u(
\end{aligned}
$$

Man rechne aus:

$$
\begin{aligned}
& \frac{1}{2}\left[\left(t_{1}+u_{1}\right)^{2} h_{u}-2\left(2 t_{1}-u_{1}\right) F+3 \mathcal{f}\right]=\mathfrak{P} \\
& {\left[2 F-\left(t_{1}+u_{1}\right) h_{u}\right]=Q} \\
& {\left[F+\left(t_{1}+u_{1}\right) h_{u}\right]=R .}
\end{aligned}
$$

Dann lautet die Gleichung (35a):

$$
\begin{aligned}
& \mathrm{P}=Q \cdot \Delta t-R \cdot \Delta u \\
& \Delta u=\frac{Q \cdot \Delta t-\mathrm{P}}{R} .
\end{aligned}
$$

und nun erhält man aus ( $34 \mathrm{~b})$ und $(35 \mathrm{~b})$ :

$$
A t=\frac{\mathfrak{D} \cdot R+\mathfrak{D} \cdot H}{Q \cdot H-E \cdot R} \ldots
$$




\section{Schlußbemerkungen.}

Bei der Berechnung einer großen Staumauer ist es unerläßlich, daß man die Rechnungsgrößen übersichtlich in Tabellenform entwickle. Sonst ist es ganz unmöglich, das Einschleichen von Fehlern zu verhüten, und es wird viel kostbare Zeit verloren. Die Berechnung des Rumpfes läßt sich mit Logarithmen und der Zimmermann'schen Rechentafel durchführen, die des Fußes dagegen kaum ohne eine vollkommene Rechenmaschine ${ }^{1}$ ).

Das kleinst mögliche Profil - wie es die vorstehende Berechnung liefert - ist zugleich das sicherste, weil dabei die größte Wahrscheinlichkeit besteht, daß die Annahmen, welche man der Berechnung zugrunde legt, auch zutreffen.

Man erzielt einen Körper von gleicher Festigkeit, indem man, entsprechend dem Gesetze, nach welchem die Beanspruchung über den Querschnitt sich verteilt, verschieden zementreiche Mörtelmischungen anwendet. Hierdurch wird zugleich die größtmögliche Kostenersparnis erreicht.

1) Ich bediene mich der Rechenmaschine von W. T. Odhner in St. Petersburg. 
Eine Schirmmauer bietet noch den besonderen Vorteil, daß man die Krone der Staumauer in der Ebene des höchsten Seestandes anlegen kann. Zum Schutze gegen überschlagende Wellen - die ja auch von den Schächten abgehalten werden müssen - führt man die Schirmmauer auf die erforderliche Höhe über den Wasserspiegel empor.

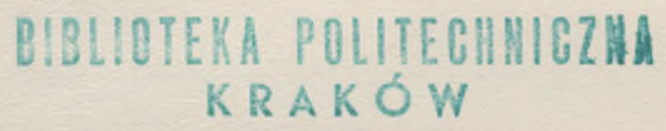




\title{
Die
}

\section{Erdbewegung bei Ingenieurarbeiten}

unter besonderer Berücksichtigung der ausftihrlichen

Vorarbeiten sowie der Abrechnung für Trassierung

von Straßen, Eisenbahnen und anderer Verkehrswege.

\author{
Von \\ Karl Allitsch,
}

Ingenieur, k. k. Professor in Innsbruck, emer. Oberingenieur und beh. autor. $\mathrm{u}$. beeid. Geometer.

Mit 10 Abbildungen im Text.

Kart. Preis M. 1.50.

In dem vorliegenden Buche beleuchtet der Verfasser die Ungenauigkeit der jetzt üblichen Näherungsmethoden und die Fehler, welche nach der alten Methode (Mittelung zweier Profile und Multiplikation ihrer Längenentfernuug) gemacht werden und weist nach, daß allein das zeichnerische Rechnen richtige Resultate ergibt. Die Schrift können wir allen Kollegen, welche mit Trassierungen oder auf Bauabteilungen beschäftigt werden, zur Anschaffung und Benutzung warm empfehlen.

(Zeitschrift des Vereins der Eisenbahnlandmesser.)

Der Verfasser, Herr Prof. K. Allitsch, vertritt in seiner Schrift die graphische Rechnungsmethode und hofft nun durch die erstere die Verfechter der analytischen Berechnungsweise für sich zu gewinnen. Ich bin der vollen Überzeugung, daß ihm dies gelingen wird, denn jeder Fachmann wird beim Studium des vorliegenden Werkchens die Überzeugung gewinnen, daß die in demselben vorgeführte Methode die vorteilhafteste auch bei den kompliziertesten Erdkörpern sein wird. Die Ausstattung der Ausgabe ist eine sehr gute und der Preis ein niedriger.

(Deutsche Techniker-Zeitung.)

\section{Elektrische Kraftbetriebe und Bahnen. Zeitsehrift} für das gesamte Anwendungsgebiet elektrischer Triebkraft. Herausgeber: Dr.-Ing. Walter Reichel, Professor an der Kgl. Technischen Hochschule Berlin-Charlottenburg. Jährl. 36 Hefte mit etwa 800 Abbildungen und zahlreichen Tafeln.

Preis für den Jahrgang M. 16.-; halbjährl. M. 8.-

Das Programm der Zeitschrift umfaßt nicht nur elektrische Eisenbałnen im gewöhnlichen Sinne des Wortes, also Vollbahnen, Nebenbahnen, Kleinbahnen und Straßeubahnen, sondern erstreckt sich auch auf Drahtseilbahnen, Massengüterbewältigung, Hebezeuge, Aufzüge und Fördermaschinen, Selbst fahrer, Schiffahrt, elektrische Treidelei und diejenigen Berg- und Hüttenwerke sowie Maschinenbetriebe, welche sich des elektrischen Stromes als Triebkraft bedienen.

\section{Journal für Gasbeleuchtung und verwandte Be-}

leuchtungsarten sowie für Wasserversorgung. Organ des Deutschen Vereins von Gas- u. Wasserfachmännern. Herausgeber und Chef-Redakteur: Geh. Hofrat Dr. H. Bunte, Prof. a. d. techn. Hochschule in Karlsruhe, General-Sekretär d. Vereins. Jährlich 52 Heite.

Preis für den Jahrgang M. 20.-; halbjährlich M. 10.- 
Journal fil Gasbeleuchtung und Wasserversorgung.

Das sJournal für Gasbeleuchtung und verwandte Beleuchtungsarten sowie für Wasserversorgung «, Organ des Deutschen Vereins von Gas- und Wasserfachmännern, steht nun in seinem 52. Jahrgange. Es behandelt nicht nur die Kohlengasbeleuchtung und Wasserversorgung in ihrem ganzen Umfange, sondern gibt auch eingehende Informationen über die verwandten Beleuchtungsarten, Azetylen, Petroleum, Spiritusglühlicht, Luftgas sowie elektrische Beleuchtung. Auch die Hygiene wird in gebührender Weise berücksichtigt. Das sJournal für Gasbeleuchtung und verwandte Beleuchtungsarten = ist auf diesem Gebiete unbestritten das erste und führende Organ. Es bedarf keiner weiteren Empfehlung.

\section{Gesundheits-Ingenieur. Zeitschrift für die gesamte Städte-}

hygiene. Organ der Vereinigung der Verwaltungsingenieure des Heizungsfaches. Herausgeg. von E. v. Böhmer, Reg.-Rat im Kais. Patentamt, Prof. Dr. Dunbar, Direktor des Staatl. Hyg. Inst. zu Hamburg, Geh. Reg.-Rat Herm. Harder, Berlin, Geh. Reg.-Rat Prof. Proskauer, Dir. d. Untersuchungsamtes f. hyg. u. gewerbl. Zwecke d. Stadt Berlin, K. Schmidt, Stadtbauinspektor, Vorstand d. Bauinsp. f. Heiz- u. Lüftungswesen d. Stadt Dresden. Die Zeitschrift erscheint wöchentlich und kostet jährlich M. 20.-; halbjährlich M. 10.-

Das Programm des im 32. Jahrgang erscheinenden "GesundheitsIngenieurs", Zeitschrift für die gesamte Städtehygiene, umfaß̉ die Gebiete : Wasserversorgung und alle mit ihr verknüpften Aufgaben, die Städtereinigung einschließlich des Kanalisationswesens, Abwrsserbeseitigung und -Reinigung, die ganze Straßenhygiene, das Abdeckereiwesen und Leichenwesen, die Fragen der Volksernăhrung und Nahrungsmittelkontrolle einschließlich des Schlachthauswesens, alle Fragen der Wohnungsbauhygiene und Baupolizei, Lüftung, Heizung, Beleuchtung, Rauchplage, Bäder, Krankenhauswesen, die Fragen der Schulhygiene und des öffentlichen Kinderschutzes, des Schutzes gegen Seuchen einschließlich Desinfektion, der Gewerbehygiene und des Feuerlöschwesens sowie noch manche andere in das Gebiet der Städtehygiene fallende Fragen; besonders auch $\mathrm{Be}$ schreibungen und Darstellungen ausgeführter oder projektierter Anlagen, Berichte über Betriebsergebnisse usw.

\section{Zeitschrift für das gesamte Turbinenwesen. Dampe-} turbinen und Dampfturbinenkraftwerke (einschl. Sehiffsturbinen, Abdampfrerwertung in Niederdruckturbinen, Kondensationsanlagen, Turbodynamos etc.) Wasserturbinen und Kraftanlagen mit Wasserturbinen $=$ Kreiselpumpen (Wasserhaltungsanlagen etc.) $=$ Kreiselgebläse, Turbokompressoren (einsehl. Gasturbinen). Unter ständiger Mitwirkung hervorragender Autoritäten herausgegeben von Prof. E. Josse, Charlottenburg. Jährl. 36 Hefte zu je 16 Seiten Text, mit zahlreichen Textabbildungen. Preis pro Jahrg. M. 18.-; halbjährl. M. 9.-.

In der $>$ Zeitschrift für das gesamte Turbinenwesen * gelangen zur Veröffentlichung wissenschaftliche Aufsätze - Theorie wie Praxis - aus dem Gebiete der Dampfturbinen (Thermodynamik) mit Einschluß der Turbodynamos, der Wasserturbinen (gesamte technische Hydraulik), der Turbinenschiffe, Wind-, Heißluft- und Gasturbinen sowie auch der Pumpen und -Ventilatoren einschließlich der rotierenden Kompressoren; sodann eingehende Beschreibung und Darstellung ausgeführter oder projektierter Anlagen, Berichterstattung über Betriebsergebnisse, Ausführungen, Projekte, Besprechung der Fachliteratur usw. Besondere Beachtung und Pflege findet auch die wirtschaftliche Seite des Turbinenbetriebes. 



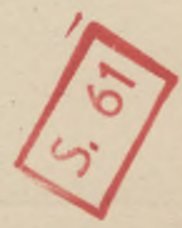





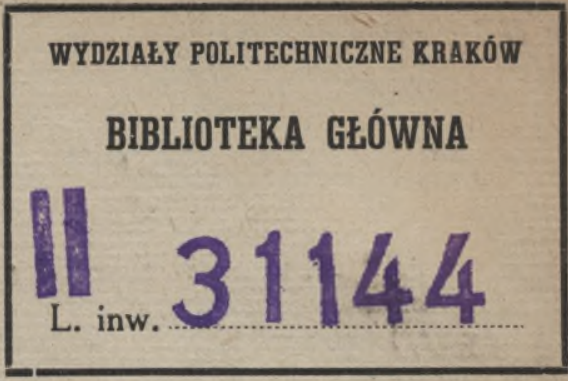

Kdn., Czapskich 4 - 678. 1. XII. 52. 10.000

Biblioteka Politechniki Krakowskiej 